\title{
FEASTING, RITUAL PRACTICES, SOCIAL MEMORY, AND PERSISTENT PLACES: NEW INTERPRETATIONS OF SHELL MOUNDS IN SOUTHERN CALIFORNIA
}

\author{
Lynn H. Gamble
}

\begin{abstract}
Shell mounds have not been investigated as prominent ritual features in southern California, despite evidence to the contrary. The largest extant shell mound in the region is on Santa Cruz Island, measures 270 by $210 \mathrm{~m}\left(44,532 \mathrm{~m}^{2}\right.$ in area), is $8 \mathrm{~m}$ higher than the terrace it rests on, is covered with 50 house depressions, and dates to 6000-2500 B.P. In the 1920s, three cemeteries were excavated at the top of El Montón; one young woman stood out among the over 200 individuals in that she was buried with 157 stone effigies. Analysis of multiple lines of evidence, including stratigraphic profiles of features, 85 radiocarbon dates, ground penetrating radar, and mortuary data, supports my claim that the mound was a persistent place where early visitors had significant feasts, constructed dwellings, buried their dead, and performed ceremonies where select groups of infants, children, and adults were revered. These mortuary rites conveyed the symbolic power of the place and created a history of events that became part of a mythical and real past that was repeatedly visited, modified, and (re)interpreted as social relationships were reinforced. This study supports the idea that shell mounds are socially constructed landscapes, not just accumulations of refuse.
\end{abstract}

A pesar de la evidencia existente, los conchales no han sido estudiados como elementos rituales prominentes en el sur de California. El conchal más grande de la región se encuentra en la isla Santa Cruz: mide 270 por 210 metros $\left(44,532 \mathrm{~m}^{2}\right.$ en área), tiene una elevación de 8 metros por encima de la terraza donde descansa, está cubierto por 50 depresiones dejadas por viviendas y se puede fechar entre 6000 y 2500 a.P. En la década de 1920, tres conjuntos de entierros fueron excavados en la cima de El Montón; una mujer joven destacó entre los más de 200 individuos debido a que fue enterrada con 157 efigies de piedra. El análisis de múltiples líneas de evidencia, incluyendo perfiles estratigráficos, 85 fechados radiocarbónicos, información de radar de penetración terrestre y datos funerarios sustenta la interpretación que el montículo constituyó un lugar duradero donde desde épocas tempranas los visitantes celebraron significativos banquetes, construyeron viviendas, enterraron a sus muertos, y realizaron ceremonias donde fueron venerados grupos selectos de infantes, niños y adultos. Estos rituales mortuorios expresaron el poder simbólico del lugar y crearon a partir de estos eventos una historia que se convirtió en parte de un pasado mítico y real. La historia fue repetida, modificada y (re)interpretada a medida que se reforzaban las relaciones sociales. Este estudio sustenta la idea de que los conchales son paisajes socialmente construidos y no únicamente acumulaciones de desechos.

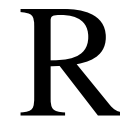

ecent investigations of shell mounds associated with hunter-gatherer-fishers are generating new interpretations and considerable debate. Questions about function and meaning of mounds, intentionality of their construction, and their symbolism are shifting discussions from viewing shell mounds in ecological terms of subsistence, resource exploitation, and seasonality to considering them as significant structures on the landscape that serve as places for daily practices and rituals that are inex- tricably tied to social memory (Thompson 2010). Human memory is socially constituted, where mythical principles are mapped as reminders of catastrophes and triumphs in the past (Knapp and Ashmore 1999:13), as an enduring record of past lives (Ingold 1993:152-153). Through memory of place and the reuse and reinterpretation of it, landscape is connected to the identity of its inhabitants (Gamble and Wilken 2008). Landscape as identity is related to collective recognition of places, often associated with symbolic or

Lynn H. Gamble - Department of Anthropology, University of California, Santa Barbara CA 93106, USA (gamble@anth.ucsb.edu)

American Antiquity 82(3), 2017, pp. 427-451

Copyright (C) 2017 by the Society for American Archaeology

doi:10.1017/aaq.2017.5 
ceremonial practices. Social memories are shaped by cumulative economic, social, and political factors (Climo and Cattell 2002) that are closely tied to the landscape. These places have the capacity to trigger self-reflection and memories of past times, people, and events (Basso 1996). They may be repeatedly visited, modified, and interpreted, often reinforcing social relationships (Pauketat 2008) and creating links to the past and the ancestors (Meskell 2003, 2007; Yoffee 2007). Memory of the past is preserved in these links, negotiated, reinterpreted, and commemorated in rituals and public events, some associated with the deceased, others with more quotidian activities; practices of remembrance emerge from repeated actions and performances (Meskell 2007:224).

\section{Mounds as Places of Persistence and Social Memory}

The materialization of social memory, whether through mortuary ceremonies, ritual congregations, or daily practices (Bourdieu 1977), provides the archaeologist with the possibility of identifying remembrance. This is not straightforward, especially for those who work in more ephermal sites associated with hunter-gatherers. Closely linked to ideas of social memory and landscape is the concept of place-making (Basso 1996) and "persistent places," locales that were used and occupied repeatedly over long periods of time (Kidder and Sherwood 2016; Schneider 2015; Thompson 2010; Thompson and Pluckhahn 2010).

Herein I present a case study from a shell mound at the far western end of Santa Cruz Island in southern California. I propose that multiple lines of evidence support the idea that El Montón (CA-SCRI-333) was a persistent place that over thousands of years of occupation became a prominent feature on the landscape, as the site became higher and expanded as people practiced rituals, staged feasts, buried their dead, and constructed houses. Before examining in detail, I first turn to discussions of shell mounds outside of southern California as theoretical and contextual background.

\section{Southeastern United States}

Several recent publications on hunter-gatherer shell mounds as places of social memory-as socially constructed monuments or landscapes, not just accumulations of refuse-are centered on sites in the southeastern United States (Kidder 2011; Marquardt 2010; Randall 2011; Russo 1994; Sassaman and Randall 2012). Many interpretations rely on detailed construction histories of mounds that include geophysical surveys such as ground-penetrating radar (GPR) (e.g., Thompson and Andrus 2011; Thompson and Pluckhahn 2010). Some investigate how mounds were tied to ritual behavior associated with feasting and mortuary ceremonialism, while others consider orientation and siting (Randall 2011; Sassaman and Randall 2012). The earliest mounds in the southeastern United States are particularly relevant to this discussion because of their similarities to El Montón. One of the most prominent Archaic sites in the southeast, Watson Brake, consists of 11 earthen mounds elliptically arranged into a 280 $\times 370 \mathrm{~m}$ complex (Saunders et al. 2005). It is one of several Archaic mound complexes in the lower Mississippi River valley that date to 5,6005,000 years ago and required planning, perhaps a reflection that non-egalitarian structures existed in the Archaic (Sassaman 2010).

\section{Other World Regions}

In the Torres Straits Islands, ceremonial mounds of dugong bones are documented, as well as mounds of large gastropods that were often used in rituals (David and Badugal 2006; McNiven 2012). Earth and shell mounds in Australia and New Zealand number in the hundreds and have been interpreted primarily in economic terms and as significant boundary markers that denote ownership (Bailey and Flemming 2008; Brockwell 2006; Cribb 1991). Hausmann and Meredith-Williams (2016) investigate middens in Saudi Arabia to explore accumulation rates of shell deposits. In Brazil, some are huge (up to $50 \mathrm{~m}$ in height), serve funerary purposes, and are prominent landscape features (Gaspar et al. 2008). Many hunter-gatherer mounds, including some in which funerary rituals are regularly reenacted, can also be found in South Africa (Jerardino 2010), Japan (Okada 1998), the Northwest 


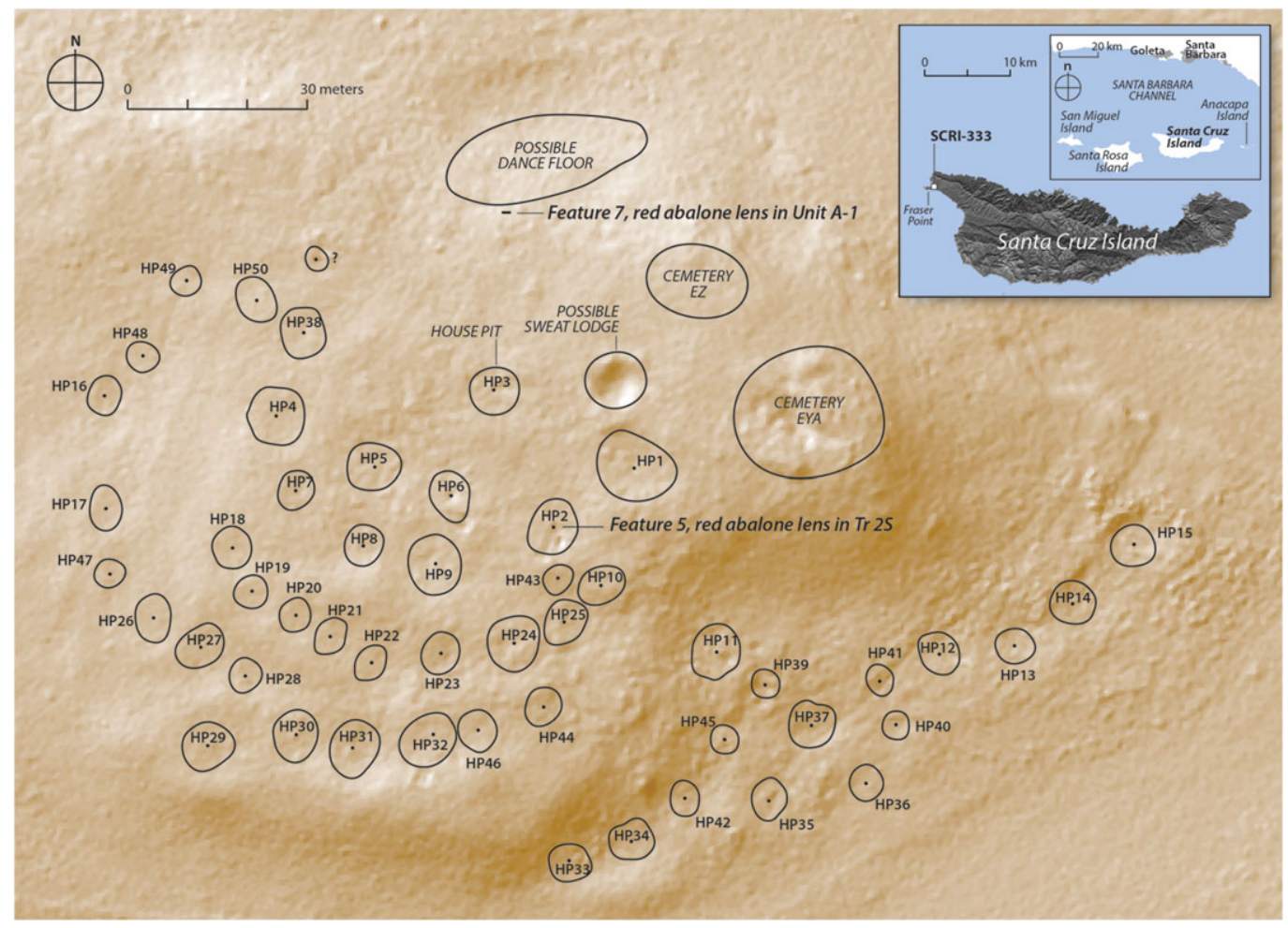

Figure 1. Map of SCRI-333. (Color online)

coast (Grier 2014; Mathews 2014), and the San Francisco Bay area.

San Francisco Bay area shell mounds are especially well-known in California, in part because of their massive size and frequency of occurrence. Many were used intensively during the Middle period (500 B.C.-A.D. 900) (Lightfoot 1997; Luby and Gruber 1999), although some became places of refuge after European colonization (Schneider 2015). Some were probably associated with polities and intentionally constructed, others served as territorial symbols and centers for numerous activities, including rituals, mortuary feasting, and places to bury the dead (Lightfoot 1997; Lightfoot and Luby 2002; Luby and Gruber 1999).

\section{Santa Barbara Channel Region}

Shell mounds in southern California have been investigated, but largely through a different theoretical lens. One on the Northern Channel Islands purportedly occupied continuously for 3,000 years is Prisoner's Harbor (CA-SCRI240). Four $\mathrm{m}(13 \mathrm{ft})$ deep in the center, it was approximately $122 \times 46 \mathrm{~m}(400 \mathrm{ft} \times 150 \mathrm{ft})$ in size (Rogers 1929:306). One feature at the site, interpreted as a feasting event, dates to the historic period (Noah 2005:280). Although mounds have been investigated on the northern Channel Islands (Braje et al. 2014), primarily through the perspective of subsistence, nothing like the mound at Prisoner's Harbor has been identified in the region except for El Montón at CA-SCRI-333.

Situated on Santa Cruz Island (Figure 1), El Montón is the best-preserved archaeological shell mound from the Middle Holocene in the Santa Barbara Channel region. With its many house depressions, features, and mortuary information, it is an ideal location to investigate the significance of a place of social memorya persistent place on the landscape, within the context of hunter-gatherer-fishers throughout the world. This project is significant because the mound was first used 6,000 years ago, is the largest existing shell mound in southern California, has numerous features, including 50 house depressions visible on the surface, 


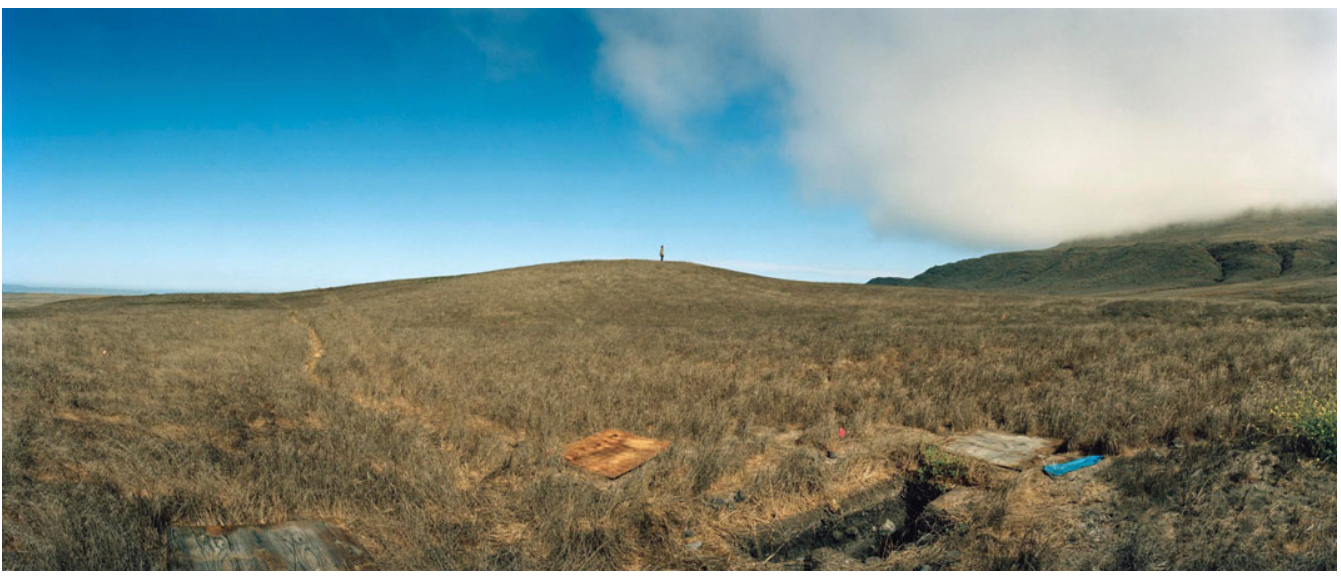

Figure 2. Person on top of El Montón (photograph by Macduff Everton). (Color online)

and has a well-documented bioarchaeological data.

\section{The Chumash Indians of Southern California}

The Chumash Indian inhabitants of the Santa Barbara Channel region exhibited a number of characteristics at European contact that are associated with complex hunter-gatherers, including the use of shell bead currencies, craft specialization, sedentism, high population densities, food storage, inherited leadership positions, and social hierarchy (Gamble 2008; Kennett 2005). Subsistence was primarily based on shellfish, fish, marine mammals, birds, and plant foods, including edible seeds, corms, and bulbs. Houses were domed-shaped with thatched roofs and often clustered in rows with pathways between them (Gamble 1995). DNA, linguistic, osteological, and archaeological evidence suggests that no major population replacements occurred during the last 7,000-10,000 years in the area (Erlandson 1994; Golla 2011; Johnson and Lorenz 2006:33), making this region ideal for exploring longterm historical trajectories of hunter-gathererfishers.

Although the Chumash had great antiquity in the Santa Barbara Channel area, they were not culturally static (e.g., Erlandson 1994; Glassow et al. 2007; Kennett 2005; King 1990). Many suggest that environmental change played a critical role in the development of sociopolitical complexity in the region about 1,000 years ago (Arnold 1992; Johnson 2000; Kennett and Kennett 2000). Less is known about societies between 6000 to 2500 B.P. than in later time periods.

This investigation is focused on the large shell mound on the western end of Santa Cruz Island, where intensive archaeological investigations have produced a rich array of radiocarbon dates within solid stratigraphic contexts, with 58 dates reported here for the first time. In this paper, I argue that El Montón was a persistent place that eventually became a significant feature on the landscape that served to create a social memory among many generations of people.

\section{Case Study: El Montón, SCRI-333}

What is most striking about El Montón is its size and prominence on the landscape (Figure 2). It is the largest extant shell mound and one of the best-preserved Early Period sites in the Santa Barbara Channel region, with most dates at the site falling between 6000 and 2500 B.P. The mound is $8-10 \mathrm{~m}$ higher than the marine terrace it rests on and measures 270 by $210 \mathrm{~m}$, approximately 4.5 ha. Over $3 \mathrm{~m}$ of deposits on the mound are cultural. Ground-penetrating radar (GPR) and archaeological excavations indicate it was built on a small natural knoll identified as the Pleistocene surface (Gamble and Simms 2016). Approximately 50 depressions between 5-13 $\mathrm{m}$ in diameter are visible on the surface, 
Table 1. Cemeteries Excavated at SCRI-333.

\begin{tabular}{llc}
\hline Excavator/Date & \multicolumn{1}{c}{ Time Period } & Number of Burials \\
\hline Olson 1928/1929 & Early Period, Phase Eya, 6000-5000 B.P. & 57 \\
Van Valkenburgh 1933/1934 & Early Period, Phase Eyb, 5000-3000 B.P. & 132 \\
Olson 1928/1929 & Early Period, Phase Ez, 3000-2600 B.P. & 48 \\
Total & & 237 \\
\hline
\end{tabular}

more than any site in the region. Excavations in five house depressions uncovered complex stratigraphic deposits, including red abalone and whalebone features that are over 5,500 years old, a large rock oven, and burned house deposits. A prominent feature on the landscape, CA-SCRI333 is visible from sites over $10 \mathrm{~km}$ away on Santa Cruz and Santa Rosa Islands. The location of El Montón was naturally defensive, with a clear view of people coming by sea or land, and ideal for a population that focused on marine resources. The adjacent Forney's Cove is the most protected harbor on the western shores of Santa Cruz Island. Identified as the only primary village site on Santa Cruz Island during the Middle Holocene (Kennett 2005:129-134), CASCRI-333 served as a central place for social, economic, and ritual activities.

\section{Previous Archaeological Investigations}

Olson (1930) conducted the earliest welldocumented excavations at CA-SCRI-333 in 1927-1928 in two cemeteries and Structure 1, where he found nearly $2 \mathrm{~m}(6 \mathrm{ft})$ of cultural deposits. He uncovered 57 burials from the earlier cemetery (6,000-5,000 B.P.) and 48 from the later component (3000-2600 B.P.; Table 1). Although Olson (1930) only briefly published on the site, he kept relatively detailed field notes, recording burial lots, grave goods, sex, age, position, orientation, and depth.

Both King (1990) and Glassow (2004) published on beads and other artifacts from Olson's excavations, but neither was a comprehensive study of the mortuary assemblage. Sholts (2010) reexamined the human remains from the site, recording age and sex of individuals.

In 1932, Richard Van Valkenburgh excavated 132 burials (over 100 complete articulated skeletons and 32 unassociated crania) in a third cemetery that spanned the period between those
Olson excavated (Table 1), but unfortunately left limited notes (Santa Barbara Museum of Natural History, "Archaeological Excavations on Frazier Point, Santa Cruz Island, California, 1932"). AMS dates from teeth of six individuals from this cemetery range between 5300-4190 cal B.P. (Monroe et al. 2010).

Finally, Wilcoxon (1993) conducted major excavations on house depressions and adjacent refuse deposits at SCRI-333 in the 1980s. Except for a brief publication (Wilcoxon 1993), he never completed his analysis.

\section{Recent Archaeological Investigations}

This paper discusses my archaeological investigations at CA-SCRI-333 between 2009 and 2016. One goal was to determine the occupational history of the mound, its formation processes, and its meaning. A detailed chronology of the site, achieved through excavation of selected house depressions, recovery of chronologically sensitive artifacts, and collection of radiocarbon samples within stratigraphic context, was integral to achieving this goal.

Therefore, I mapped 50 house depressions (Figure 1), many of which are in rows that appear to be on purposely constructed terraces on the mound (Figure 3). Detailed stratigraphic profiles of trenches were made, with radiocarbon samples identified on them (Figures 4 and 5). Three house depressions (Structures 2, 6, and 32) had features (Lens $\mathrm{C}$ ) that were identified as probable burned house deposits (Figure 4). Two additional depressions lacked this lens, but one had a large burned-rock feature in its center (Figure 5b). I also analyzed notes and collections from the two cemeteries (Collection of Manuscripts from the Archaeological Archives of the Phoebe A. Hearst Museum of Anthropology, \# 442, Original Field Notebooks and Photo Prints of Santa Barbara Mainland and Santa Cruz Island Excavations, 


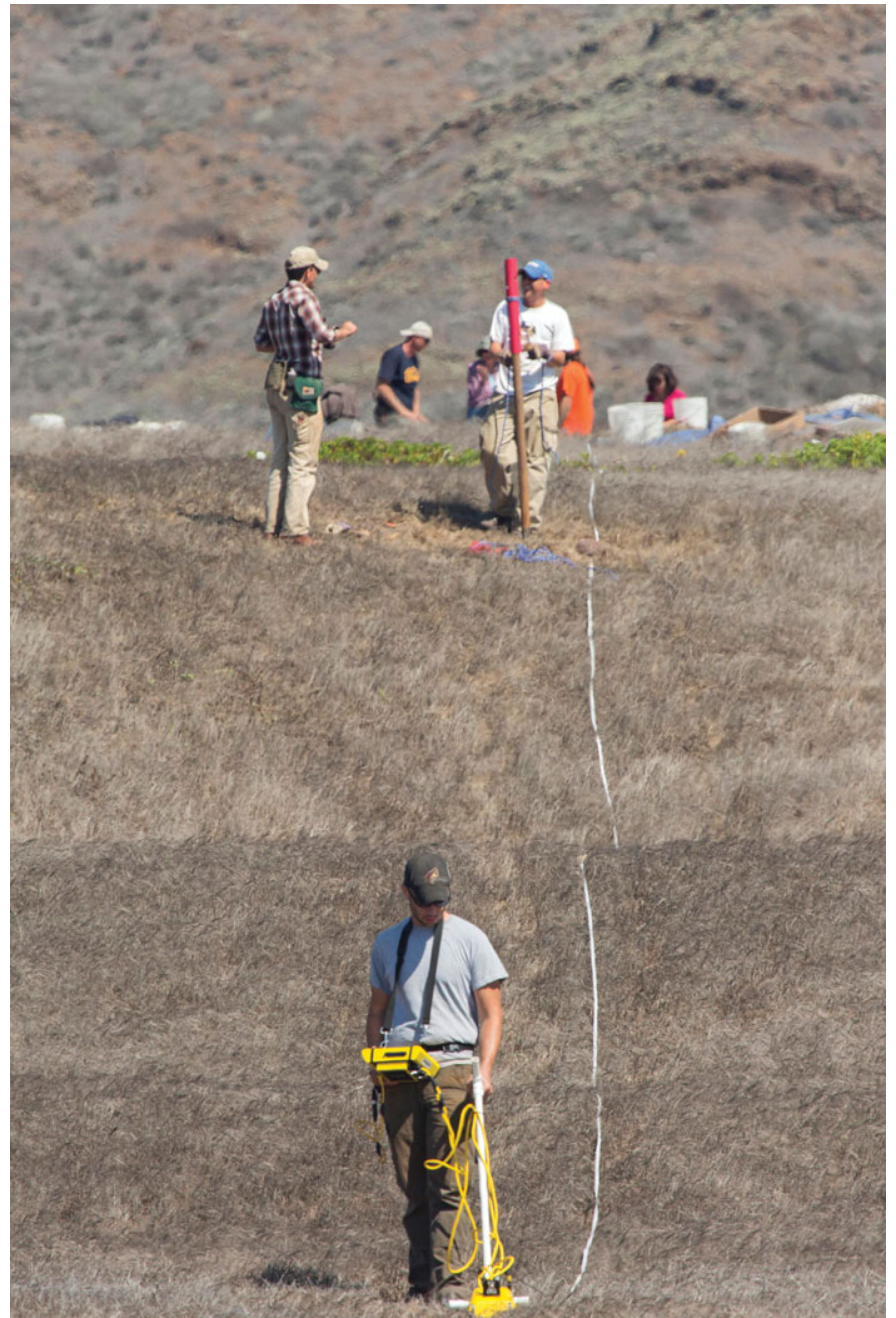

Figure 3. Photograph showing terraces at SCRI-333 (photograph by Macduff Everton). (Color online)

Including Some Quad Sheets with Site Locations [by R. L. Olson, 1927-1928]) and identified Olson's excavation units in the field.

\section{Results of Current Analysis from Old and New Investigations at SCRI-333}

\section{El Montón, a Persistent Place}

How and why did the site of El Montón become a place of such significance during the Early Period? I suspect that the location was originally an attraction because of the rocky intertidal zones, fresh water sources, kelp beds, sandy beaches, and cove for boats. It also commands an impressive view of Santa Rosa Island and the west end of Santa Cruz Island and the surrounding hills — significant for defensive reasons and also as a prominent place on the landscape. I propose that over time, as people visited the site, they eventually settled there, constructing thatch-covered houses and harvesting a wealth of marine resources adjacent to the site. They chose the highest part of the mound to bury their dead in rites that included endowing some who died with grave goods that distinguished them from others. Undoubtedly inherent in these rites were significant ceremonies and feasts. Other occa- 

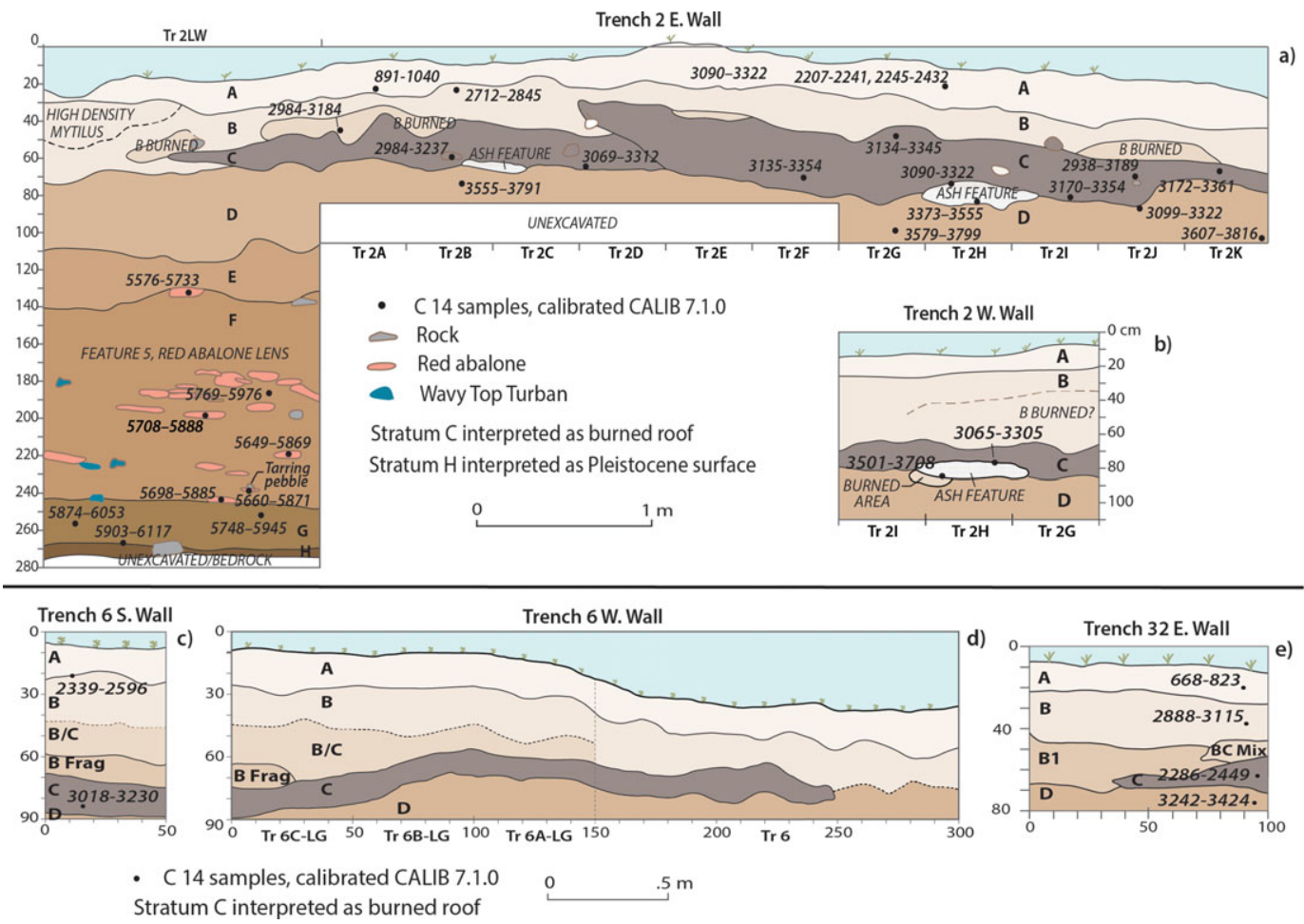

Figure 4. (a) Stratigraphic profile with AMS dates for Trench 2 east wall, (b) stratigraphic profile with AMS dates for Trench 2 west wall, ash feature, (c) and (d) stratigraphic profiles with AMS dates for Trench 6, south and west walls, (e) stratigraphic profile with AMS dates for Trench 32, east wall. (Color online)

sions, such as calendrical events like the Winter Solstice, were also probably associated with feasts. Eventually, the mound became higher and more expansive-a prominent place on the landscape that could be viewed from the sea and many surrounding settlements.

\section{Chronology}

Eighty-five radiocarbon dates have been analyzed from SCRI-333 (Table 2); I obtained 69 of these 85 dates, most from shell and in clear stratigraphic context (Figures 4 and 5), 58 of which are reported here for the first time. The calibrated dates range between $668-5117$ cal B.P., with all but four (95 percent) dating between 2207-6117 cal B.P. (Table 2), and are consistent with most chronologically sensitive artifacts from the site. The four dates that postdate $2207 \mathrm{cal}$ B.P. are from the upper $30 \mathrm{~cm}$ of the site, and probably a result of people visiting later in time, not site inhabitants.

\section{Remembering the Dead}

Three cemeteries excavated in the 1920s and 1930s are immediately adjacent to each other and situated at the highest point on the mound. None of the house depressions visible at the site are in the cemetery region, suggesting that the Chumash inhabitants recognized this area as formal sacred space. Their choice of the summit probably had significant symbolic meaning, as decisions of where to place the dead are not usually based on functional expediency, but instead often have powerful meanings about social geography (Parker Pearson 2000). In some societies, the dead still inhabit the world as spirits, and cemeteries can be viewed as a liminal space between the living and spirit world (Meskell 2007). When standing on the mound's apex, the view of the sea, Santa Rosa Island, San Miguel, and the west end of Santa Cruz Island are striking, yet visibility is limited to only certain portions of the site itself, thereby situating that space as private and prominent at the same 
Unit A-1 North Wall

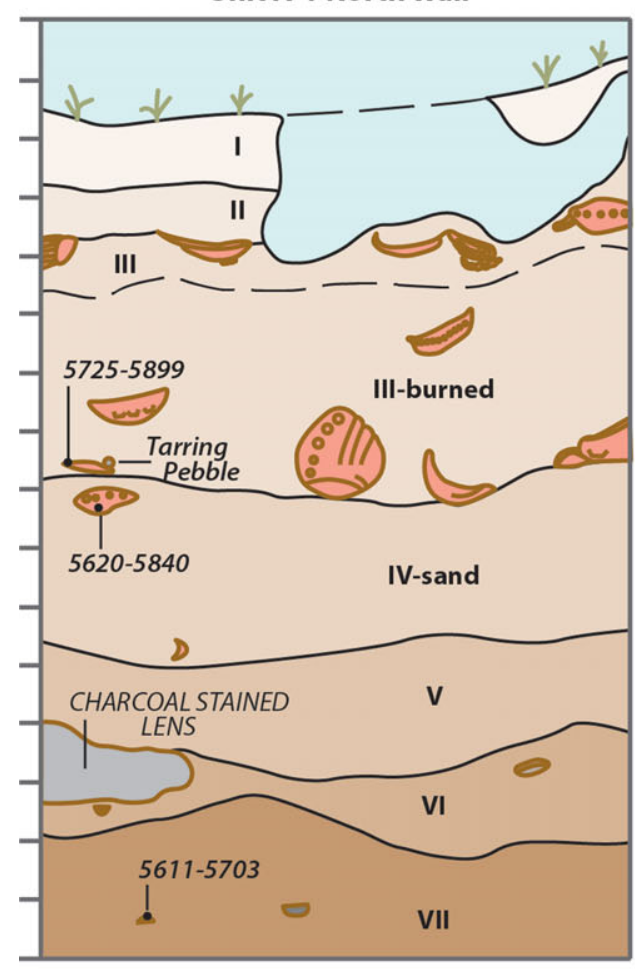

Unit A-1 South Wall

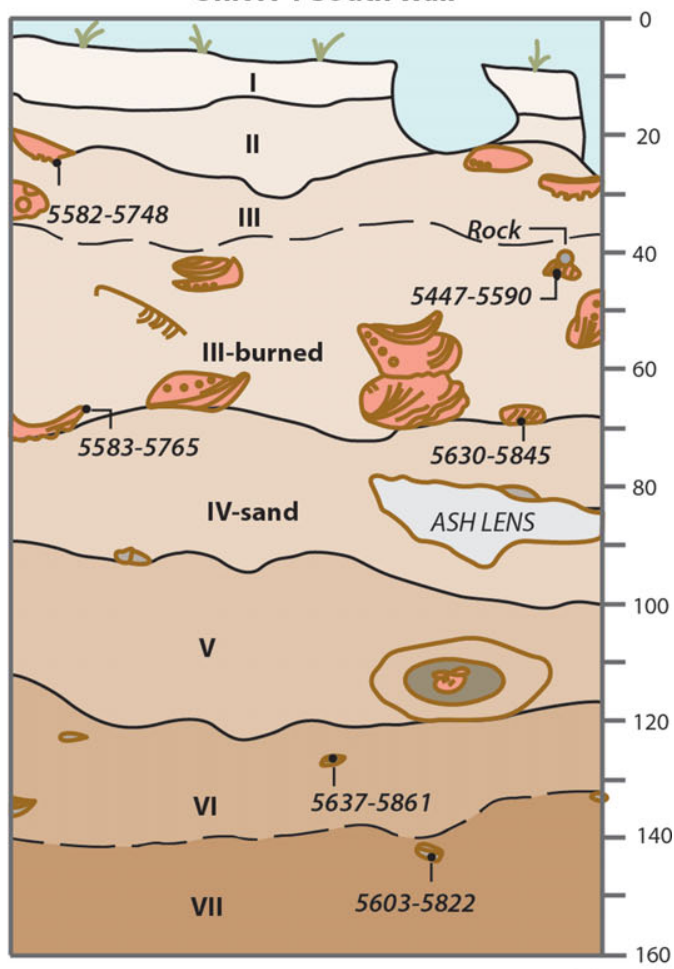

(a)

- C 14 samples, calibrated CALIB 7.1.0 Dashed line = difficult to discern stratigraphic change

- Rock

$\odot$ Red abalone

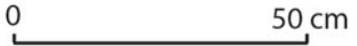

\section{Unit 14 East Wall}

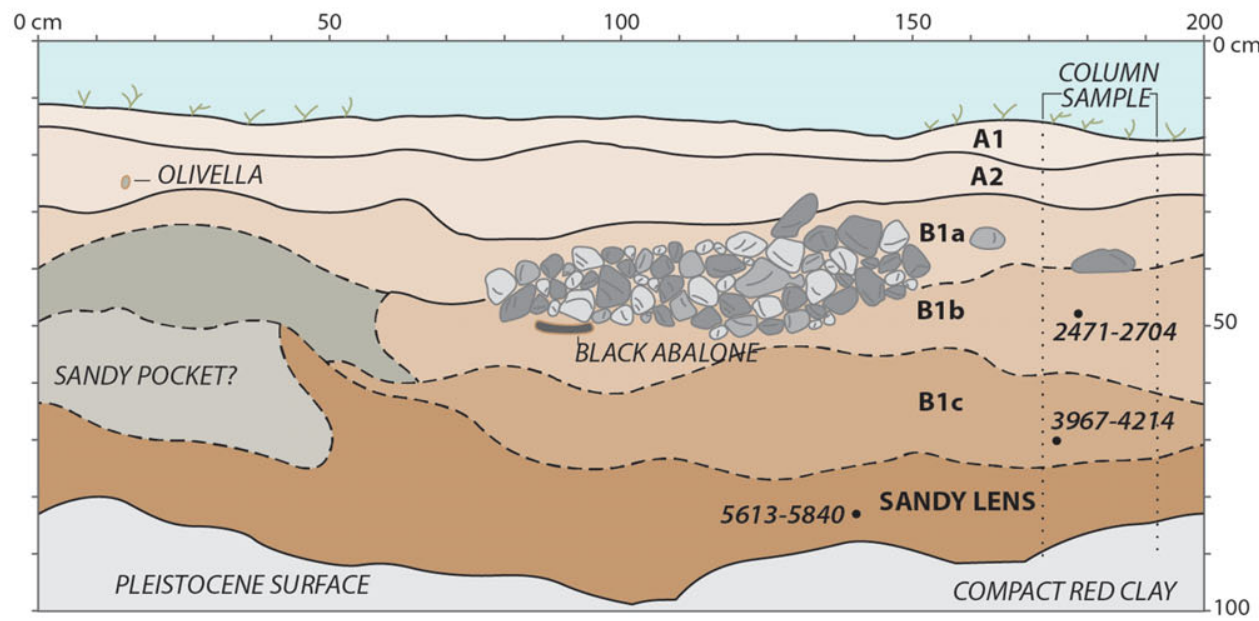

(b)

Figure 5. (a) Stratigraphic profile with AMS dates for Unit A-1, north and south walls, (b) stratigraphic profile with AMS dates for Unit 14, east wall. (Color online) 
Table 2. Radiocarbon Dates at SCRI-333.

\begin{tabular}{|c|c|c|c|c|c|c|c|c|c|}
\hline Lab \# & Unit/Trench & Depth $(\mathrm{cm})$ & Strata & Feature & Material & $\begin{array}{l}\text { Conventional } \\
{ }^{14} \text { C Age (B.P.) }\end{array}$ & $2 \sigma \mathrm{cal} \mathrm{B.P.}$ & $2 \sigma$ cal B.P. Median & Reference \\
\hline D-AMS 1389 & Trench 2A & 21 & $\mathrm{~A}$ & Above structure & M. californianus & $1666 \pm 23$ & $891-1040$ & 951 & New \\
\hline D-AMS 13310 & Trench $2 \mathrm{H}$ & 22 & A & Above structure & M. californianus & $2899 \pm 25$ & $\begin{array}{l}2207-2241 \\
2245-2432\end{array}$ & 2325 & New \\
\hline UCIAMS- 94055 & $\begin{array}{l}\text { Trench } \\
32 \mathrm{~A} / \mathrm{B}\end{array}$ & 20 & A & Above structure & M. californianus & $1465 \pm 20$ & $668-823$ & 741 & $\begin{array}{c}\text { Jazwa et al. } \\
2013\end{array}$ \\
\hline UCIAMS- 94051 & Trench 6C & 21 & A & Above structure & M. californianus & $3000 \pm 20$ & $2339-2596$ & 2443 & $\begin{array}{c}\text { Jazwa et al. } \\
2013\end{array}$ \\
\hline D-AMS 1383 & Trench 2B & 21 & B & Above structure & M. californianus & $3260 \pm 24$ & $2712-2845$ & 2768 & New \\
\hline D-AMS 13311 & Trench $2 \mathrm{H}$ & 37 & $\mathrm{~B}$ & Above structure & M. californianus & $2980 \pm 29$ & $\begin{array}{l}2318-2573 \\
2579-2581 \\
2589-2591\end{array}$ & 2419 & New \\
\hline UCIAMS- 94056 & $\begin{array}{l}\text { Trench } \\
\text { 32A/B }\end{array}$ & 34 & B & Above structure & M. californianus & $3460 \pm 20$ & $2888-3115$ & 3000 & $\begin{array}{c}\text { Jazwa et al. } \\
2013\end{array}$ \\
\hline UCIAMS- 87889 & Trench 6A & 29 & B & Above structure & Charcoal & $2485 \pm 20$ & $\begin{array}{l}2489-2645 \\
2647-2715\end{array}$ & 2586 & $\begin{array}{c}\text { Jazwa et al. } \\
2013\end{array}$ \\
\hline D-AMS 1384 & Trench 2A & 42 & B burned & Above structure & M. californianus & $3511 \pm 29$ & $2948-3184$ & 3071 & New \\
\hline D-AMS 13312 & Trench 2J & 55 & B burned & Above structure & M. californianus & $3611 \pm 29$ & 3094-3329 & 3209 & New \\
\hline D-AMS 1390 & Trench 11 & 70 & B Lower & & H. rufescens & $5428 \pm 29$ & $5449-5596$ & 5530 & New \\
\hline D-AMS 10985 & Trench $2 \mathrm{~K}$ & 67 & $\mathrm{~B} / \mathrm{B}$ burned & Above structure & M. californianus & $3664 \pm 25$ & $3172-3361$ & 3274 & New \\
\hline UCIAMS- 87891 & Trench 6B & 49.5 & $\mathrm{~B} / \mathrm{C}$ & Above structure & Charcoal & $2540 \pm 20$ & $\begin{array}{l}2506-2530 \\
2536-2590 \\
2616-2633 \\
2697-2745\end{array}$ & 2715 & $\begin{array}{c}\text { Jazwa et al. } \\
2013\end{array}$ \\
\hline D-AMS 1393 & Unit 14B & 48 & $\mathrm{~B} 1 \mathrm{~b}$ & & M. californianus & $3093 \pm 24$ & $2471-2704$ & 2600 & New \\
\hline D-AMS 1391 & Unit 14B & 71 & $\mathrm{~B} 1 \mathrm{c}$ & & M. californianus & $4318 \pm 29$ & $3967-4214$ & 4087 & New \\
\hline D-AMS 1385 & Trench 2B & 55 & $\mathrm{C}$ & Burned structure & H. rufescens & $3547 \pm 32$ & $2984-3237$ & 3118 & New \\
\hline D-AMS 13309 & Trench 2D & 55 & $\mathrm{C}$ & Burned structure & M. californianus & $3595 \pm 28$ & $3069-3312$ & 3187 & New \\
\hline D-AMS 1382 & Trench 2F & 68 & $\mathrm{C}$ & Burned structure & M. californianus & $3642 \pm 33$ & $3135-3354$ & 3249 & New \\
\hline D-AMS 10992 & Trench 2G & 49 & $\mathrm{C}$ & Burned structure & M. californianus & $3635 \pm 29$ & $3134-3345$ & 3241 & New \\
\hline D-AMS 10991 & Trench $2 \mathrm{H}$ & 75 & $\mathrm{C}$ & Slightly darker C & M. californianus & $3606 \pm 30$ & $3090-3322$ & 3202 & New \\
\hline D-AMS 10993 & Trench $2 \mathrm{H}$ & 76 & $\mathrm{C}$ & Burned structure & M. californianus & $3590 \pm 26$ & $3065-3305$ & 3180 & New \\
\hline D-AMS 10988 & Trench 2I & 80 & $\mathrm{C}$ & Burned structure & M. californianus & $3658 \pm 22$ & $3170-3354$ & 3268 & New \\
\hline D-AMS 10987 & Trench 2J & 69 & $\mathrm{C}$ & Burned structure & M. californianus & $3507 \pm 33$ & $2938-3189$ & 3065 & New \\
\hline
\end{tabular}


Table 2. Continued.

\begin{tabular}{|c|c|c|c|c|c|c|c|c|c|}
\hline $\mathrm{Lab} \#$ & Unit/Trench & Depth $(\mathrm{cm})$ & Strata & Feature & Material & $\begin{array}{l}\text { Conventional } \\
{ }^{14} \text { C Age (B.P.) }\end{array}$ & $2 \sigma$ cal B.P. & $2 \sigma$ cal B.P. Median & Reference \\
\hline UCIAMS- 87888 & $\begin{array}{l}\text { Trench } \\
\text { 32A/B }\end{array}$ & 60 & $\mathrm{C}$ & Burned structure & Charcoal & $2510 \pm 20$ & $\begin{array}{l}2494-2598 \\
2610-2639 \\
2682-2732\end{array}$ & 2583 & $\begin{array}{c}\text { Jazwa et al. } \\
2013\end{array}$ \\
\hline UCIAMS- 94053 & $\begin{array}{l}\text { Trench } \\
32 \mathrm{~A} / \mathrm{B}\end{array}$ & 63 & $\mathrm{C}$ & Burned structure & M. californianus & $2920 \pm 20$ & $2286-2449$ & 2344 & $\begin{array}{c}\text { Jazwa et al. } \\
2013\end{array}$ \\
\hline UCIAMS- 87892 & Trench 6C & 61.5 & $\mathrm{C}$ & Burned structure & Charcoal & $2970 \pm 20$ & $\begin{array}{l}3071-3185 \\
3190-3209\end{array}$ & 3136 & $\begin{array}{c}\text { Jazwa et al. } \\
2013\end{array}$ \\
\hline UCIAMS- 94052 & Trench 6C & 84 & $\mathrm{C}$ & Burned structure & M. californianus & $3555 \pm 20$ & $3018-3230$ & 3130 & $\begin{array}{c}\text { Jazwa et al. } \\
2013\end{array}$ \\
\hline D-AMS 10986 & Trench 2J & 85 & $\mathrm{C}$ at $\mathrm{D}$ & Burned structure & H. cracherodii & $3608 \pm 24$ & $3099-3322$ & 3205 & New \\
\hline D-AMS 10994 & Trench $2 \mathrm{H}$ & 84 & ash feature & Hearth?, bottom & M. californianus & $3955 \pm 25$ & $3501-3708$ & 3609 & New \\
\hline D-AMS 10990 & Trench $2 \mathrm{H}$ & 85 & ash feature & Hearth?, top & M. californianus & $3834 \pm 25$ & $3373-3555$ & 3464 & New \\
\hline D-AMS 1381 & Trench 2B & 72 & $\mathrm{D}$ & Below structure & M. californianus & $3993 \pm 30$ & $3555-3791$ & 3656 & New \\
\hline D-AMS 10999 & Trench 2G & 74 & $\mathrm{D}$ & Below structure & Charcoal & $3132 \pm 28$ & $\begin{array}{l}3251-3303 \\
3322-3405 \\
3427-3443\end{array}$ & 3359 & New \\
\hline D-AMS 10989 & Trench 2G & 99 & $\mathrm{D}$ & Below structure & M. californianus & $4009 \pm 24$ & $3579-3799$ & 3676 & New \\
\hline D-AMS 11000 & Trench $2 \mathrm{H}$ & $80-90$ & $\mathrm{D}$ & Below structure & Charcoal & $2967 \pm 22$ & $3067-3209$ & 3130 & New \\
\hline D-AMS 10984 & Trench $2 \mathrm{~K}$ & 103 & $\mathrm{D}$ & Below structure & M. californianus & $4030 \pm 23$ & $3607-3816$ & 3706 & New \\
\hline UCIAMS- 94054 & $\begin{array}{l}\text { Trench } \\
\text { 32A/B }\end{array}$ & 75 & $\mathrm{D}$ & Below structure & M. californianus & $3720 \pm 20$ & $3242-3424$ & 3340 & $\begin{array}{c}\text { Jazwa et al. } \\
2013\end{array}$ \\
\hline UCIAMS- 87890 & Trench 6A & 78 & $\mathrm{D}$ & Below structure & Charcoal & $3080 \pm 20$ & $3234-3359$ & 3292 & $\begin{array}{c}\text { Jazwa et al. } \\
2013\end{array}$ \\
\hline D-AMS 1386 & Trench 2LW & 138 & $\mathrm{~F}$ & Red abalone lens & H. rufescens & $5555 \pm 30$ & $5576-5733$ & 5648 & New \\
\hline D-AMS 1388 & Trench 2S & 218 & $\mathrm{~F}$ & Red abalone lens & H. rufescens & $5570 \pm 34$ & $5575-5769$ & 5664 & New \\
\hline D-AMS 1387 & Trench 2S & 220 & $\mathrm{~F}$ & Red abalone lens & H. rufescens & $5646 \pm 31$ & $5649-5869$ & 5758 & New \\
\hline D-AMS 11001 & Trench 2S & $190-200$ & $\mathrm{~F}$ & Red abalone lens & Charcoal & $5085 \pm 26$ & $\begin{array}{l}5749-5830 \\
5844-5909\end{array}$ & 5811 & New \\
\hline D-AMS 11002 & Trench 2S & $190-200$ & $\mathrm{~F}$ & Red abalone lens & Charcoal & $5095 \pm 30$ & $\begin{array}{l}5749-5830 \\
5844-5914\end{array}$ & 5813 & New \\
\hline D-AMS 10974 & Trench 2S & 185 & F5 & Red abalone lens & M. californianus & $5766 \pm 28$ & $5769-5976$ & 5891 & New \\
\hline D-AMS 10977 & Trench 2S & 195 & F6 & Red abalone lens & H. rufescens & $5683 \pm 24$ & $5708-5888$ & 5800 & New \\
\hline D-AMS 10978 & Trench 2S & 237 & F8 & Red abalone lens & H. rufescens & $5654 \pm 28$ & $5660-5871$ & 5769 & New \\
\hline D-AMS 10975 & Trench 2S & 242 & F9 & Red abalone lens & H. rufescens & $5675 \pm 24$ & $5698-5885$ & 5792 & New \\
\hline
\end{tabular}


Table 2. Continued.

\begin{tabular}{|c|c|c|c|c|c|c|c|c|c|}
\hline Lab \# & Unit/Trench & Depth $(\mathrm{cm})$ & Strata & Feature & Material & $\begin{array}{l}\text { Conventional } \\
{ }^{14} \text { C Age (B.P.) }\end{array}$ & $2 \sigma \mathrm{cal}$ B.P. & $2 \sigma$ cal B.P. Median & Reference \\
\hline D-AMS 1392 & Unit 14B & $37-40$ & FAR feature & FAR feature & M. californianus & $3019 \pm 25$ & 2349-2616 & 2480 & New \\
\hline D-AMS 10973 & Trench 2S & 237 & G1 & Red abalone lens & M. californianus & $5750 \pm 26$ & $5748-5945$ & 5874 & New \\
\hline D-AMS 10972 & Trench 2S & 256 & $\mathrm{G} 2$ & Red abalone lens & Urchin & $5824 \pm 25$ & $5874-6053$ & 5946 & New \\
\hline D-AMS 10976 & Trench $2 \mathrm{~S}$ & 267 & G3 & Red abalone lens & M. californianus & $5869 \pm 28$ & 5903-6117 & 5998 & New \\
\hline D-AMS 10971 & Trench 2S & 268 & G3 & Red abalone lens & M. californianus & $5766 \pm 30$ & $5766-5977$ & 5890 & New \\
\hline D-AMS 13316 & Unit A-2 & 22 & I & & M. californianus & $3984 \pm 24$ & $3549-3761$ & 3644 & New \\
\hline D-AMS 10995 & Trench 11 & 23 & & & M. californianus & $3503 \pm 29$ & $2939-3175$ & 3060 & New \\
\hline D-AMS 13317 & Unit A-2 & 30 & II & & M. californianus & $4198 \pm 27$ & $3825-4049$ & 3922 & New \\
\hline D-AMS 13315 & Unit A-2 & 35 & II & & M. californianus & $3930 \pm 26$ & $3472-3678$ & 3578 & New \\
\hline D-AMS 10998 & Unit A-1 & 42.5 & III & Red abalone lens & H. rufescens & $5479 \pm 25$ & $5481-5649$ & 5582 & New \\
\hline D-AMS 13314 & Unit A-2 & 53 & III & & M. californianus & $4334 \pm 26$ & $3984-4224$ & 4111 & New \\
\hline D-AMS 10979 & Unit A-1 & 20 & III & Red abalone lens & H. rufescens & $5574 \pm 26$ & $5582-5748$ & 5666 & New \\
\hline D-AMS 10997 & Unit A-1 & 43 & III burned & Red abalone lens & H. rufescens & $5423 \pm 28$ & $5447-5590$ & 5526 & New \\
\hline D-AMS 10983 & Unit A-1 & 66 & III burned & Red abalone lens & H. rufescens & $5581 \pm 26$ & $5583-5765$ & 5673 & New \\
\hline D-AMS 10980 & Unit A-1 & 67 & III burned & Red abalone lens & H. rufescens & $5621 \pm 24$ & $5630-5845$ & 5720 & New \\
\hline D-AMS 10982 & Unit A-1 & 75 & III burned & Red abalone lens & H. rufescens & $5703 \pm 25$ & $5725-5899$ & 5817 & New \\
\hline D-AMS 10996 & Trench 11 & 88 & & & M. californianus & $4109 \pm 24$ & $3749-3862$ & 3805 & New \\
\hline D-AMS 10981 & Unit A-1 & 80 & IV? & Red abalone lens & H. rufescens & $5615 \pm 26$ & $5620-5840$ & 5712 & New \\
\hline D-AMS 1394 & Unit 14B & 83 & Sand & & M. californianus & $5613 \pm 29$ & $5613-5840$ & 5710 & New \\
\hline D-AMS 13306 & Unit A-1 & 126 & VI & & M. californianus & $5633 \pm 31$ & $5637-5861$ & 5739 & New \\
\hline D-AMS 13307 & Unit A-1 & 142 & VII & & M. californianus & $5605 \pm 31$ & $5603-5822$ & 5701 & New \\
\hline D-AMS 13308 & Unit A-1 & 153 & VII & & H. cracherodii & $5568 \pm 31$ & $5611-5703$ & 5661 & New \\
\hline D-AMS 13313 & Unit A-2 & 96 & VII & & M. californianus & $4628 \pm 26$ & 4404-4615 & 4503 & New \\
\hline Beta-35005 & Trench 2 & $30-40$ & & & Marine Shell & $4130 \pm 70$ & $3635-4048$ & 3834 & $\begin{array}{c}\text { Breschini et al. } \\
\text { 1996:56 }\end{array}$ \\
\hline CAMS-9099 & Unit 11B & $200-210$ & & & M. californianus & $4240 \pm 60$ & $3808-4172$ & 3982 & $\begin{array}{l}\text { Kennett } \\
\text { 1998:462 }\end{array}$ \\
\hline CAMS-9098 & Unit 11B & $200-210$ & & & M. californianus & $4300 \pm 60$ & $3871-4251$ & 4062 & $\begin{array}{l}\text { Kennett } \\
1998: 462\end{array}$ \\
\hline CAMS-9100 & Unit 11B & $200-210$ & & & M. californianus & $4300 \pm 70$ & $3851-4280$ & 4063 & $\begin{array}{l}\text { Kennett } \\
\text { 1998:462 }\end{array}$ \\
\hline CAMS-9097 & Unit 11B & $200-210$ & & & M. californianus & $4370 \pm 60$ & $3972-4364$ & 4164 & $\begin{array}{l}\text { Kennett } \\
1998: 462\end{array}$ \\
\hline
\end{tabular}


Table 2. Continued

\begin{tabular}{|c|c|c|c|c|c|c|c|c|c|}
\hline Lab \# & Unit/Trench & Depth (cm) & Strata & Feature & Material & $\begin{array}{l}\text { Conventional } \\
{ }^{14} \text { C Age (B.P.) }\end{array}$ & $2 \sigma$ cal B.P. & $2 \sigma$ cal B.P. Median & Reference \\
\hline CAMS-9661 & Unit 11B & $210-220$ & & Red abalone lens & M. californianus & $5580 \pm 60$ & $5570-5853$ & 5684 & $\begin{array}{l}\text { Kennett } \\
\text { 1998:462 }\end{array}$ \\
\hline CAMS-9660 & Unit 11B & $210-220$ & & Red abalone lens & M. californianus & $5610 \pm 90$ & $5541-5919$ & 5721 & $\begin{array}{l}\text { Kennett } \\
1998: 462\end{array}$ \\
\hline UCR-1956 & Unit 11B & $70-80$ & & & Charcoal & $4015 \pm 100$ & $4235-4826$ & 4504 & $\begin{array}{l}\text { Wilcoxon } \\
\text { 1993:148 }\end{array}$ \\
\hline UCR-1955 & Unit 11B & $10-30$ & & & Charcoal & $2160 \pm 100$ & $\begin{array}{l}1903-1908 \\
1924-2350\end{array}$ & 2155 & $\begin{array}{l}\text { Wilcoxon } \\
\text { 1993:148 }\end{array}$ \\
\hline UCR-1951 & Unit 2B-S & $20-30$ & & & Charcoal & $2700 \pm 70$ & $2722-2963$ & 2819 & $\begin{array}{l}\text { Wilcoxon } \\
\text { 1993:148 }\end{array}$ \\
\hline UCR-1952 & Unit 2B-S & $90-100$ & & & Charcoal & $3330 \pm 90$ & $\begin{array}{l}3378-3732 \\
3743-3775 \\
3789-3826\end{array}$ & 3569 & $\begin{array}{l}\text { Wilcoxon } \\
\text { 1993:148 }\end{array}$ \\
\hline UCR-1957 & Unit 32B & $10-20$ & & & Charcoal & $2300 \pm 90$ & $\begin{array}{l}2066-2081 \\
2108-2542 \\
2561-2618 \\
2631-2701\end{array}$ & 2320 & $\begin{array}{l}\text { Wilcoxon } \\
\text { 1993:148 }\end{array}$ \\
\hline UCR-1530 & Unit 6A & 220 & & Red abalone lens & H. rufescens & $5190 \pm 135$ & $4885-5560$ & 5237 & $\begin{array}{l}\text { Wilcoxon } \\
\text { 1993:148 }\end{array}$ \\
\hline UCR-1954 & Unit 6A & $120-130$ & & & Charcoal & $3310 \pm 70$ & $3386-3695$ & 3541 & $\begin{array}{l}\text { Wilcoxon } \\
\text { 1993:148 }\end{array}$ \\
\hline UCR-1953 & Unit 6A & $20-30$ & & & Charcoal & $1410 \pm 95$ & $\begin{array}{l}1089-1109 \\
1128-1133 \\
1146-1159 \\
1172-1533\end{array}$ & 1328 & $\begin{array}{l}\text { Wilcoxon } \\
\text { 1993:148 }\end{array}$ \\
\hline UCR-1852 & Unit 6A & $220-230$ & & Red abalone lens & H. rufescens & $4590 \pm 95$ & $4207-4779$ & 4466 & $\begin{array}{l}\text { Wilcoxon } \\
\text { 1993:148 }\end{array}$ \\
\hline
\end{tabular}




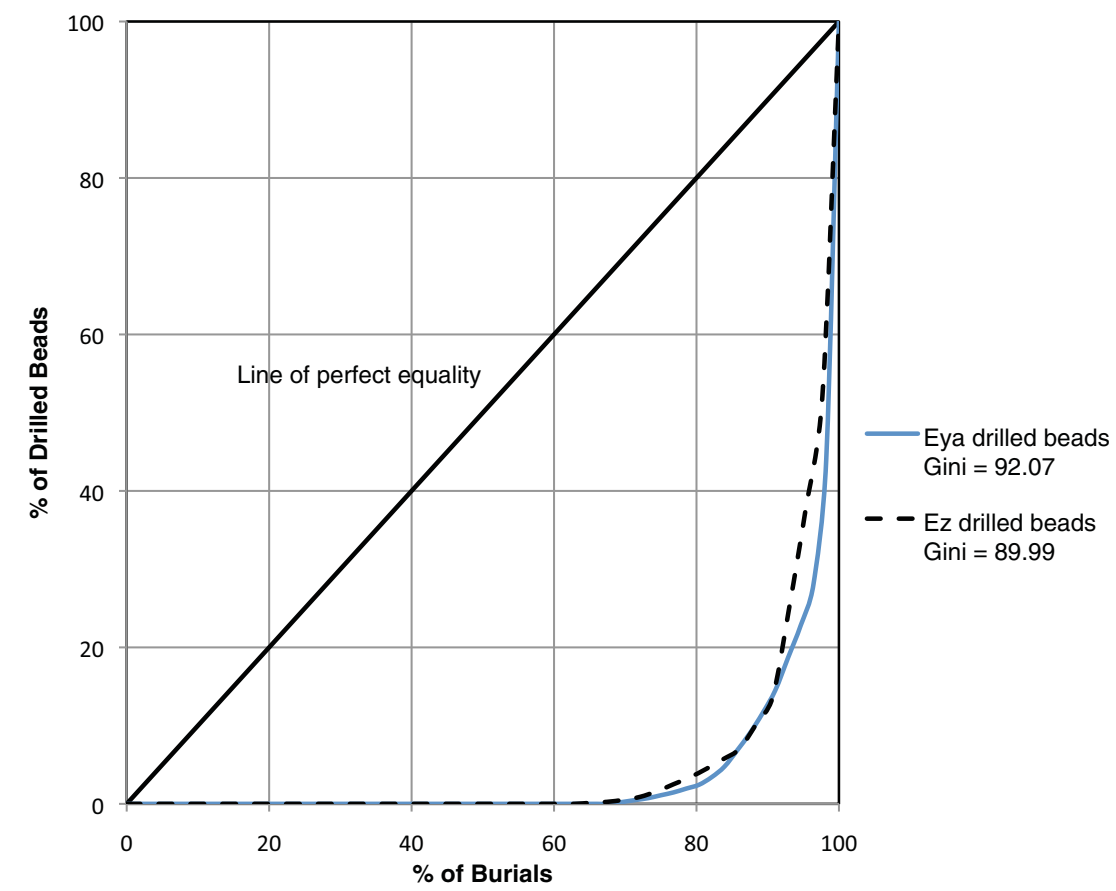

Figure 6. Lorenz curves and gini coefficient indexes of drilled beads from cemeteries Eya and Ez. (Color online)

time. Prominent places can be viewed in social contexts that help ensure rights over natural resources (Parker Pearson 2000). The prominent mound at the west end of Santa Cruz Island can be considered a liminal interface between land and sea-a transformational space where religious rituals mark the transcendence between the material and immaterial, between life and death.

Evidence that people were not treated equally in death is apparent in both cemeteries excavated by Olson. Some individuals were buried with many grave goods, and others with nothing or very few. To measure the degree of inequality in the distribution of beads, ornaments, and other grave goods, Lorenz curves and Gini Coefficients were constructed (Figure 6 and Table 3). The Gini coefficient converts the Lorenz curves to a single number between 0 and 100 percent, allowing one to numerically compare curves. A Gini coefficient of 100 percent conveys the maximum inequality. The Gini coefficients for all artifacts and the two Lorenz curves plotted for drilled beads by burial in each cemetery (Table 3 and Figure 6) indicate clear inequality.
Table 3. Gini Index for Different Types of Grave Goods.

\begin{tabular}{lcc}
\hline Item & Eya & Ez \\
\hline All grave goods & 76.19 & 78.68 \\
All beads \& ornaments & 80.16 & 79.19 \\
Drilled beads & 92.07 & 89.99 \\
Non-drilled beads & 84.01 & 84.94 \\
\hline
\end{tabular}

The earlier cemetery (Phase Eya, 6000-5000 B.P.) contrasts with the later one at CA-SCRI333 and other cemeteries in the Chumash region in the higher frequency of infants and children (41.9 percent) compared with adults (Table 4). Especially intriguing are the many subadults buried with numerous grave goods (Figure 7). For example, five of the six individuals with more than 150 beads and ornaments are infants. Although Olivella biplicata (now known as Callinax biplicata) shell beads were considered a type of currency in the Late period (A.D. 11501804), there is no clear evidence this was the case thousands of years ago. Instead, beads and ornaments were probably items of adornment associated with higher-status individuals. The 
Table 4. Percentages of Infants and Children at Five Chumash Cemeteries.

\begin{tabular}{llccr}
\hline Site & \multicolumn{1}{c}{ Time Period } & \% Infants & \% Infants and Children & Number of Burials \\
\hline SCRI-333 & Early Period Eya, 6000-5000 B.P. & 36.4 & 41.9 & 55 \\
SCRI-333 & Early Period Ez, 3000-2600 B.P. & 12.5 & 18.5 & 48 \\
Malibu LAN-264 & Middle Period 5, A.D. 950-1150 & & 17 & 90 \\
Medea Creek LAN-243 & Protohistoric, A.D. 1300-1785 & & 24 & 296 \\
Malibu LAN-264 & Historic Period, A.D. 1775-1805 & 21 & 140 \\
\hline
\end{tabular}

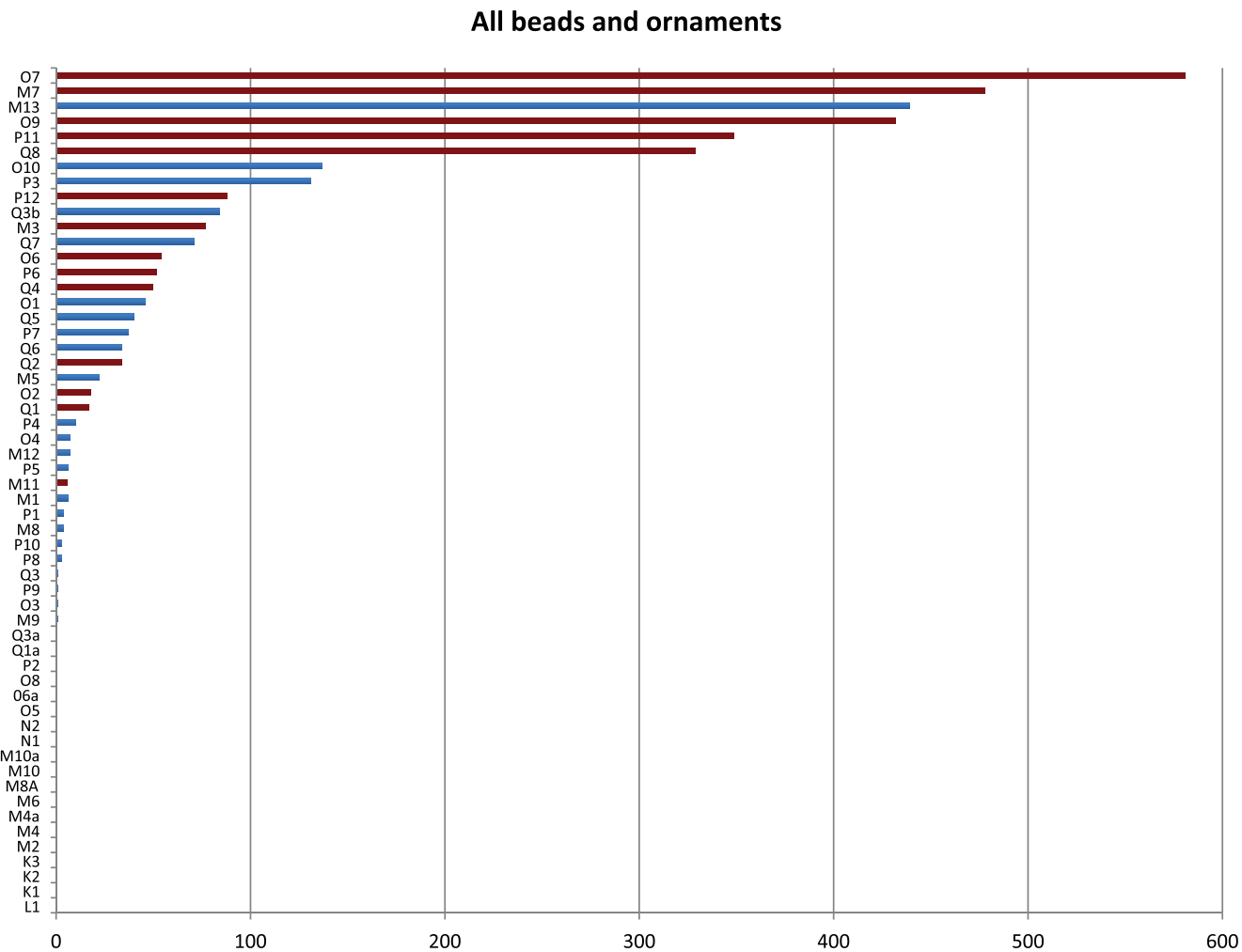

Figure 7. Graph of beads and ornaments by burial in Early Period phase Eya (6000-5000 B.P.) Cemetery at SCRI-333. (Color online)

presence of many beads with infants and children implies ascribed status, or at the very least, special treatment that others were not afforded. Other, less common, types of grave goods are listed in Table 5. The later cemetery (Phase Ez, ca. $3000-2600$ B.P.) is similar to the earlier one in that many subadults were buried with beads and ornaments, although the percentage of subadults compared to adults was much less. Significant literature about infants and children in the archaeological record has emerged over the last couple of decades (Kamp 2001; Lillehammer 2010; Sofaer Derevenski 1994); some have focused on a history of children as invisible, marginalized, and disempowered in anthropological publications (Baxter 2008). In certain regions of the world, archaeological evidence suggests that infants were perceived as incomplete persons, often not even afforded formal burial rites. We see that at CA-SCRI-333 they are treated similarly to adults, if not with even greater veneration, and were perceived as complete persons even as infants.

The central portion of the later cemetery $(\mathrm{Ez})$ is distinctive because of placement and treatment of burials, with nine individuals in extended 
Table 5. Artifacts in Cemeteries.

\begin{tabular}{|c|c|c|}
\hline Artifact Type & Eya Cemetery & Ez Cemetery \\
\hline $\begin{array}{l}\text { Shell disc or tube beads, } \\
\text { drilled }\end{array}$ & 825 & 1,563 \\
\hline Shell beads, whole & 2,822 & 7,386 \\
\hline Bone beads & 5 & 11 \\
\hline Stone beads & 9 & 185 \\
\hline Shell ornaments & 64 & 48 \\
\hline Stone ornaments & 0 & 10 \\
\hline Stone ornament/bead blanks & 0 & 3 \\
\hline Bone pins & 39 & 5 \\
\hline Bone implements & 19 & 12 \\
\hline Bone awls & 8 & 5 \\
\hline Bone barbs & 10 & 6 \\
\hline Mortars & 4 & 4 \\
\hline Pestles & 2 & 9 \\
\hline Digging stick weights & 9 & 1 \\
\hline Digging stick weight blanks & 0 & 2 \\
\hline Points & 1 & 1 \\
\hline Abalone pries & 3 & 0 \\
\hline $\begin{array}{l}\text { Asphaltum basketry } \\
\text { impressions }\end{array}$ & 20 & 189 \\
\hline Tarring pebbles & 5 & 48 \\
\hline Olivella shells, unworked & 33 & 381 \\
\hline Worked shell & 0 & 1 \\
\hline Pebbles & 19 & 30 \\
\hline Chipped stone & 18 & 40 \\
\hline Effigies & 1 & 166 \\
\hline Charmstones & 0 & 2 \\
\hline Shell dishes & 9 & 14 \\
\hline Shells with ochre & 2 & 2 \\
\hline Shell with asphaltum & 0 & 5 \\
\hline Turtle shell & 3 & 2 \\
\hline Whale bone objects & 19 & 4 \\
\hline Possible sun stick stones & 2 & 0 \\
\hline Ochre & 13 & 8 \\
\hline Steatite bowls & 2 & 1 \\
\hline Pipes & 2 & 0 \\
\hline Total & 3,968 & 10,144 \\
\hline
\end{tabular}

positions, in contrast to the flexed positions of all other burials in both cemeteries. This was not a result of chronological differences, as beads and other temporally diagnostic grave accompaniments are contemporaneous (King 1990) with those from the rest of the cemetery. Most of these nine were buried with a greater diversity and quantity of grave goods than other people, and, especially striking, these were the only individuals interred with black serpentine artifacts, including beads, ornaments, ornament blanks, and a small bowl. The serpentine in the cemetery is a hard stone that closely resembles that found near Figueroa Mountain in the San Rafael range on the mainland, about $80 \mathrm{~km}$ north of Santa Cruz Island. A most remarkable burial in this central area is an adolescent female (C7) interred with six serpentine beads. What really made her stand apart from others, however, were the associated 157 effigies (95 percent of all the effigies) (Figure 8), some of which were painted and shaped. Effigies in southern California have been interpreted as instrumental in the mobilization and control of supernatural powers (Applegate 1978). Although it is unknown exactly how they were used thousands of years ago, it is likely that this young woman was recognized as someone with special significance and ritual power. This is not unlike young women buried with effigies in the mainland Chumash site of Malibu during the Middle Period (Gamble et al. 2001).

The inhabitants of El Montón buried their dead at the top of the mound for over three millennia; ceremonies surrounding mourning and interment were undoubtedly significant events in the lives of the inhabitants of and visitors to El Montón (Arnold 2006; Meskell 2003; Parker Pearson 2000). Mortuary events have been suggested as critical in the creation of persistent places in shell mounds in Australia and the Green River Valley in Kentucky (Littleton and Allen 2007; Moore 2015). Few material remains encountered by archaeologists are as clearly sacred as mortuary space (Moore 2004).

\section{Houses and Other Features}

In addition to the cemeteries, approximately 50 house depressions, a possible dance area, and a possible sweat lodge have been documented at CA-SCRI-333, among other features. The house depressions range between $20-133 \mathrm{~m}^{2}$ in area each and are situated on a series of five terraces that radiate around the southern, western, and eastern portions of the mound (Figures 1 and 9). The largest depression (Structure 1, diameter $=$ $13 \mathrm{~m}$ ) is near the center of the mound at the highest elevation, not far from the three cemeteries. This depression had the best visibility of the surrounding settlements on Santa Cruz Island, the eastern part of Santa Rosa Island, and San Miguel Island. Hierarchy in the sizes of houses is clear (Figure 9). No occupational floors have been encountered yet. However, a highly distinctive lens (Lens C) was identified 

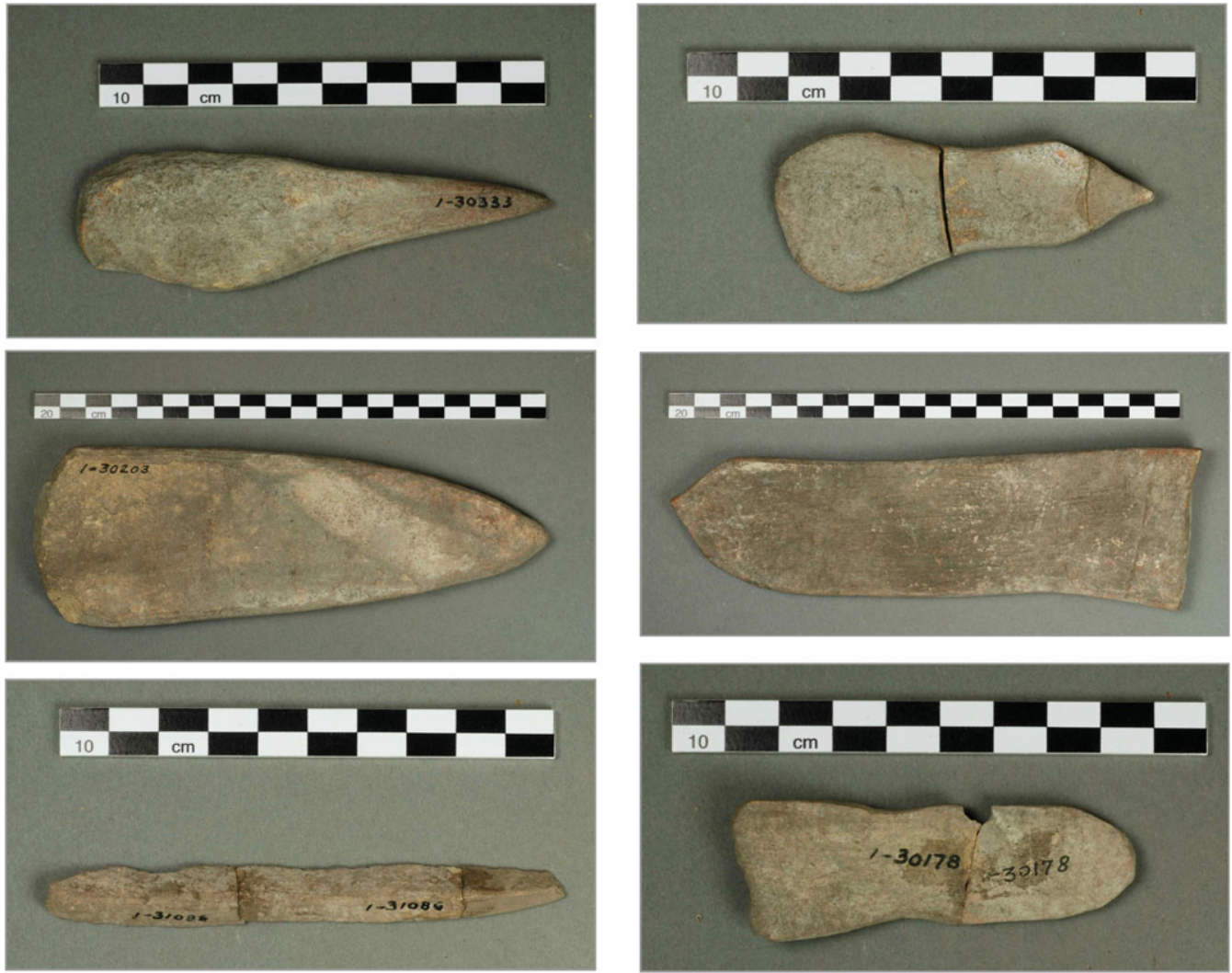

Figure 8. Sample of effigies found in burial C7, Cemetery Ez. (Color online)

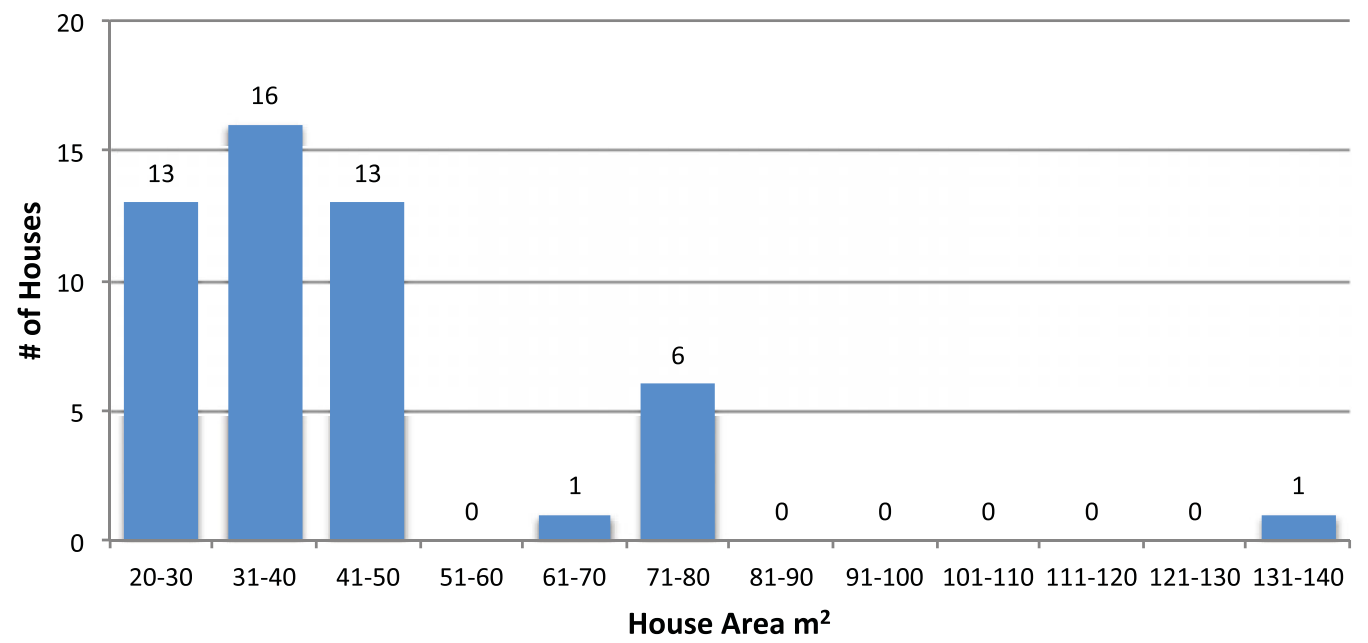

Figure 9. Distribution of house depressions by area. (Color online) 
in three house depressions, and does not occur in deposits outside of house depressions. It is not a continuous stratum (Figure 4), but a lens consisting of light-colored ash with few to no artifacts. In Trench 2, fire-reddened soil was noted in a few places immediately beneath Lens $\mathrm{C}$, suggesting that something burned in situ. This lens can be meters in length, and therefore does not appear to be a hearth, but a larger burned feature. Based on these data, I interpret Lens C as the remains of burned thatch (originally on the roofs of the structures) and possibly woven mats or sea grass that covered the floors. Most archaeological examples of domestic structures in the region lack clay floors, rock rings, and other distinguishing features, although exceptions exist (Gamble 1995).

AMS dates (Table 2 and Jazwa et al. 2013) from Lens C in Structures 2 (diameter $=10 \mathrm{~m})$, $6($ diameter $=8 \mathrm{~m})$, and $32($ diameter $=10 \mathrm{~m})$ suggest that occupation in Structures 2 and 6 overlapped (2938-3354 cal B.P. and 3018-3230 respectively). Interestingly, both are on the same terrace near the top of the mound, while Structure 32, which was apparently occupied earlier (2286-2732 cal B.P.), is situated on a lower terrace (Figure 1). Two additional dated house depressions, Structures 11 (diameter $=9 \mathrm{~m})$ and 14 (diameter $=7 \mathrm{~m}$ ), lacked Lens $\mathrm{C}$, so I am uncertain whether their occupational levels are contemporaneous. GPR results indicate possible buried houses (Gamble and Simms 2016). However, their presence needs confirmation. Because CA-SCRI-333 was occupied for 3,800 years and all dated house deposits are in the latter 3,250 years, I expect that earlier houses not visible on the surface existed.

Although Structure 14, which is situated on the lowest terrace of the mound, lacked Lens C, a large $(1 \times 1.25 \mathrm{~m})$ and impressive burned-rock feature was discovered (Figures 1 and $5 b$ ) that consisted of approximately 100 heavily burned rocks within a very dark and greasy soil with bits of charcoal. It is slightly concave in cross-section and much larger than average Chumash hearths. I propose that it was a rock oven that probably was used after the abandonment of the structure. The location of the feature was on the leeward side of the mound, which served as a natural windbreak. Roasting ovens in southern California have been used to cook yucca (Yucca whippelei), blue dicks (Dichelostemma capitatum), and pine nuts (Gamble and Mattingly 2012; Gill 2015; Timbrook 2007). Yucca does not grow on Santa Cruz Island; however, a burned-rock roasting pit with the remains of blue dicks was documented at CASCRI-619/620 (Gill 2015). No blue dicks were found in the CA-SCRI-333 feature; however, some were recovered at the site. Thick-shelled pine nuts, probably those of Torrey Pines from Santa Rosa Island, were also at the site but not associated with the feature. Torrey Pine nuts, very large and nutritious (Gamble and Mattingly 2012), are geographically closer than mainland Grey Pines, the only other California species with thick-shelled pine nuts. Torrey Pines are rare and grow only in San Diego and Santa Rosa Island. The burned-rock feature is strikingly similar in appearance to hundreds in San Diego that are interpreted as ovens for processing Torrey Pine nuts (Gamble and Mattingly 2012).

\section{Subsistence or Feasting}

Shellfish remains are ubiquitous at El Montón and make up the bulk of the midden deposits. Most not associated with red abalone features are highly fragmented and dominated by mussel (Mytilus californianus), which usually comprises between 91 and 96 percent of the species represented by weight (Landazuri 2015), followed by barnacle, black abalone, and sea urchins, with few or no red abalone. The red abalone features at the site differ significantly from other shell concentrations in that the shells are large and often whole, with red abalone consisting of 10 percent or more of the species by weight. Using multiple lines of evidence, I propose that at least one of the red abalone features may be the remains of a feasting event. I define feasting in a broad sense as communal consumption of food and or drink (Dietler and Hayden 2001) beyond daily sharing of meals. Instead, it is sharing in more atypical contexts, such as large communal meals associated with unusual occasions within or outside the context of ceremonies, which may involve singing, dancing, and other performative acts. Archaeological indicators for feasting include the presence of rare or labor-intensive plant or animal taxa, signs of wasting of food, or the presence of exceptionally large quantities 
Table 6. Weight and Percentage of Shellfish Taxa in Red Abalone Features.

\begin{tabular}{lrrrrr}
\hline & \multicolumn{2}{c}{ Trench $2 \mathrm{~S}, 40-60 \mathrm{cmbd}$} & & \multicolumn{2}{c}{ Trench A-1, 30-50 cmbd } \\
\cline { 2 - 3 } \cline { 6 - 6 } Taxa & \multicolumn{1}{c}{ Weight } & & & Weight & $\%$ \\
\hline Balanus & $1,123.2$ & 3.2 & & 269.4 & 0.8 \\
H. cracherodii & 198.7 & 0.6 & & 412.5 & 1.2 \\
H. rufescens & $4,491.4$ & 12.8 & & $28,880.1$ & 82.4 \\
Leaf barnacle & 270.2 & 0.8 & & 5.5 & 0.0 \\
Limpet & 180.0 & 0.5 & & 37.5 & 0.1 \\
Mytilus & $22,201.3$ & 63.2 & & $4,379.2$ & 12.5 \\
Urchin & $5,347.6$ & 15.2 & & 62.6 & 0.2 \\
Wavy top & $1,059.4$ & 3.0 & & 531.1 & 1.5 \\
Miscellaneous & 281.9 & 0.8 & & 492.1 & 1.4 \\
Total & $35,153.7$ & 100.0 & $35,069.8$ & 100.0 \\
\hline
\end{tabular}

of food (Hayden 2001:39-42). Rapid deposition and minimal trampling of faunal remains are also markers of feasting.

The red abalone feature (Feature 5, which is relatively close to the cemeteries) in Trench 2SLG, 110-245 cm below datum, dates between 5575-6117 cal B.P. (Figures 1 and $4 \mathrm{a}$ and Table 2), a period of time when sea surface temperatures (SSTs) were warmer (Braje et al. 2009; Kennett 2005). The red abalone feature was about $135 \mathrm{~cm}$ thick, thicker than any documented on the Channel Islands. Species of shell identified for two high-density levels consist of 12.8 percent red abalone, 15.2 percent sea urchin, and 63.2 percent mussel (Table 6), more red abalone and urchin than found in other parts of the site. Most red abalone shells were stacked (possibly purposely placed), bright in color, and whole, as were mussel, many of which had both valves attached. Remarkably, some sea urchins, a fragile shell, were also relatively intact, with large portions or whole shells found in situ.

Although the ratio of bone to shell is only 2.1:97.9 in Feature 5, those found are noteworthy. Articulated vertebrae were occasionally found in situ, illustrating little disturbance or trampling after their deposition. For example, 12 articulated leopard shark vertebrae were in Feature 5 (Figure 10), along with 20 additional leopard shark vertebrae in the same level. Another unusual characteristic were three large fragmentary bones in one level identified as baleen whales (Mysteceti), most likely gray whale (Eschrichtius robustus). The Chumash are not known to hunt whales, so their presence is probably from beached whales. Dolphin bones $(n$ $=18$ ), identified only in Feature 5 at CA-SCRI333, were similar in size and adjacent to one other, indicating limited postdepositional disturbance. Dolphin remains are relatively uncommon on Santa Cruz Island except at Punta Arena (CASCRI-109), a site situated on the south coast that overlaps in time with CA-SCRI-333; hundreds of dolphin remains were identified there dating between 5,300 and 6,300 years ago (Glassow 2005). The hunting of dolphins is often associated with seaworthy watercraft and harpoons, and some scholars consider them a high-status food (Noah 2005). Although not nearly as many dolphin remains were found at El Montón, the presence of 18 dolphin bones suggests that dolphins were either found washed up on the beach, hunted, or perhaps imported from Punta Arena. Irrespective of how they arrived at the settlement, they are rare and significantly only found in the red abalone feature. Another unusual marine species identified in Feature 5 was one Mola mola bone, the only remains of this unusual fish found at the site. Mola mola or ocean sunfish are huge, the largest of any teleost fish, can weigh in excess of 1,500 kg (Porcasi and Andrews 2001), and are often associated with warmer waters. As with the dolphin bones, Mola mola are rare at the site and found only in the red abalone feature.

Not only rare faunal remains were in the red abalone feature, but uncommon ethnobotanical remains were also. Certain taxa of plant remains found only in Feature 5 include the pits of holly-leaved cherry and manzanita, despite the fact that more liters of flotation samples were 


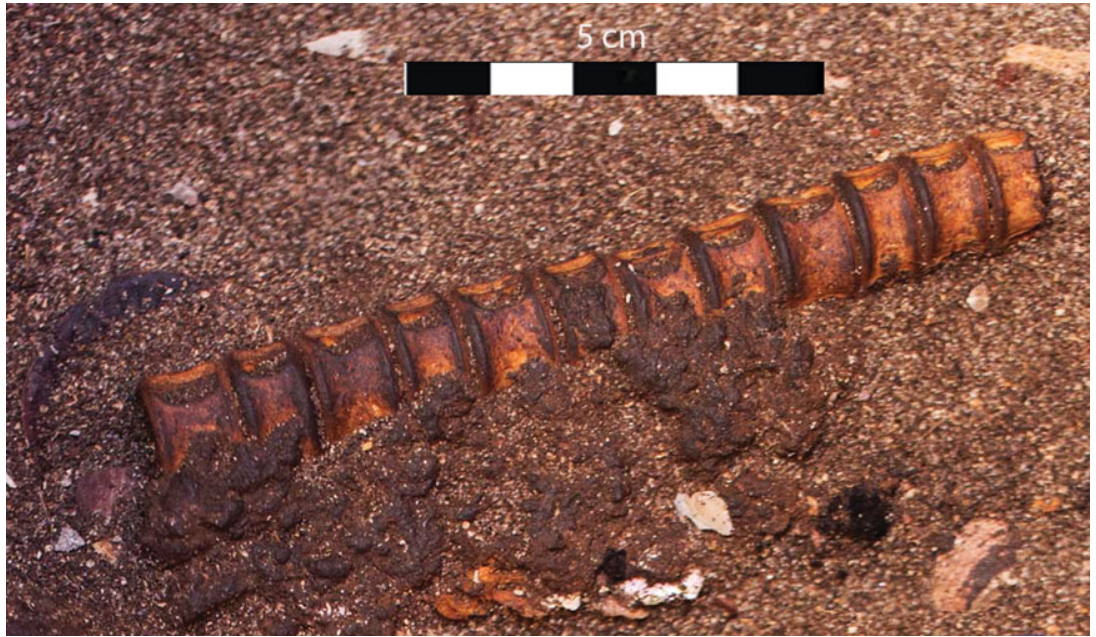

Figure 10. Articulated shark centra in red abalone feature. (Color online)

processed in other areas of the site. Holly-leaved cherry and manzanita are both important plant foods in the Chumash region. The pits of hollyleaved cherry were preferred over the fruits and required time-consuming leaching. Cherry pits were highly valued and traded between the mainland and Northern Channel Islands (Timbrook 2007). Archaeobotanical samples of them are documented in ceremonial features on San Clemente Island. Their presence provides additional evidence that Feature 5 may be the remains of a feasting event.

Another prominent red abalone feature was found in Unit A-1 (Feature 7) on the north side of the mound (Figures 1 and 5a). Although its dates, 5481-5899 cal B.P. (Table 2), are similar to those in Feature 5, it differs significantly in that the shell was not as brightly colored and was not in a dark greasy soil matrix, but instead in sand. The percentage of red abalone (82.4 percent) in this feature was significantly higher than the percentage (12.8 percent) in Feature 5 (Table 6), and matches some of the high densities observed on Santa Rosa and San Miguel Islands (Braje and Erlandson 2016; Braje et al. 2009; Glassow 2015, 2016). The red abalone feature in Unit A1 was not nearly as thick as Feature 5, (40-50 $\mathrm{cm}$ in thickness), with most of the red abalone occurring within a $20 \mathrm{~cm}$ level. Although not all bone in Feature 7 has been identified by taxa yet, relatively few bones were in the feature, with the ratio of bone to shell 0.1:99.9. Three possibly articulated dolphin vertebrae and a few tiny scattered fish bones were noted during excavation. In addition, very little charcoal compared with that in Feature 5 was recovered. Many shells were whole, but fragmented easily because they were burned, although burning did not appear to be in situ. They may have been burned elsewhere and then redeposited on the north side of the mound away from the living area. The lack of a greasy soil matrix, scarcity of bones, and lack of articulated vertebrae support the idea that this was not the remains of an in situ feasting event, but more likely redeposited shells.

The spatial distribution of red abalone provides additional clues about the nature of these features. Nineteen augers placed across the site proved especially effective, in combination with excavations of units and trenches, in the identification of buried red abalone deposits and their extent. Interestingly, red abalone features were in limited patches, versus continuous strata throughout the site. One auger a few meters from the thickest documented red abalone lens (Feature 5) had only a couple of red abalone.

In summary, at least one of the red abalone features at El Montón appears to be the result of a feasting event. First, the bright color of the shells and their intact nature, including more fragile species such as sea urchins, suggest that shells were deposited rapidly in an area of the 
site where there was little trampling afterward. Second, numerous AMS dates overlap in time, indicating rapid deposition. Third, the presence of rare species, some of which are highly valued and previously found in ceremonial features elsewhere, adds further evidence that Feature 5 was the remains of a feasting event. Finally, the patchy distribution of red abalone suggests that at least some of them are remains of events, not accretions developing over long periods of time. Not all red abalone features at the site appear to be a result of feasts. Some, such as Feature 7, may be redeposition of food remains, whereas others, especially those that are limited in thickness and lack rare species, may be more related to quotidian activities.

\section{Discussion}

My primary goal in this paper is to move beyond looking at shell mounds in southern California as primarily accumulations of refuse that inform us about subsistence and climate change, and instead to consider the significance of landscape and persistent places. This entails viewing them as locales that take on symbolic meanings as they are repeatedly occupied and as social memories are embodied through events that are collectively modified over time. This study differs significantly from many regional publications on shell middens because of its focus on social memory, persistent places, identity, and context. It is situated in theoretical underpinnings elaborated among scholars investigating hunter-gatherer mounds in the southeast and other regions. Although El Montón differs in construction form from many mounds elsewhere, significant parallels exist. The concept of persistent places is especially fitting in the current example. As in Australia and the southeastern United States, certain locales were occupied for centuries and even millennia after founding events such as mortuary rituals. The many features and mortuary data provide multiple lines of evidence to address issues of collective memory making, performative acts associated with the commemoration of the dead, ritual events, and significance of place.

Mortuary rituals actively reflect the construction of social orders among the living in memory of the dead through practices such as elaborate feasting and gift giving that serve to honor the deceased but also create important alliances between the living (Ekengren 2013; Hayden 2009; Mills and Walker 2008; Parker Pearson 2000). Objects placed with individuals are parts of complex and transformational rituals (Bell 1992) and, as such, provide symbolic meanings that may differ outside these ceremonial contexts. Ceremonies commemorating individuals are moments that will be remembered, contested, and reinterpreted over time (Mills and Walker 2008).

The Chumash at El Montón recognized the top of the mound as a sacred space, a sanctified area where, for thousands of years, they conducted rituals centered on honoring the dead. Not all those who died were treated equally; some had many more grave goods than others. Infants and children were treated very much like the adults. Claims that institutionalized social inequalities did not exist as early as 6000-2500 B.P. in the Santa Barbara Channel region are not supported by this study; instead, these data confound the concept that people were relatively equal thousands of years ago. Otherwise, why were some infants and children not afforded the same recognition at death as others, or adults? I suggest that not everyone was equal.

Especially intriguing are the burials in the central portion of Cemetery Ez at CA-SCRI333. Here we see individuals buried in extended positions, instead of flexed with rare trade items such as serpentine beads, ornaments, and a bowl. The adolescent girl buried in an extended position with 157 stone effigies clearly was treated differently than others, perhaps because of special powers she possessed. These patterns compel us to rethink this early period of timethe differential treatment of people in death most likely mirrors differential treatment in life.

El Montón differs from other sites on the northern Channel Islands in the size of the massive shell mound and its 50 visible house depressions (in addition to probable buried ones), many more than any other site. Most sites from this period have less than five visible depressions and are much smaller than CASCRI-333 (Gamble and Barbier 2015), which was most likely a center where people from 
surrounding settlements assembled for ritual gatherings, knowledge exchange, potential marriage partners, and many other reasons. It differs from some Archaic sites in southeastern United States, such as Watson Brake, in that it is not an earthen mound, does not appear to have been planned, and has evidence of residential living in addition to ceremonial features. Despite these differences, the presence alone of hunter-gatherer mounds as massive as those at Watson Brake and El Montón over 5,000 years ago challenges traditional concepts of early hunter-gatherer groups as egalitarian, generalized foragers (Sassaman and Heckenberger 2004). Instead, the mounds are testimony to transformations in the landscape and in societya reflection of greater hierarchy.

I propose that the shell mound at El Montón originally attracted maritime-oriented populations because of its ideal location, including abundant resources. Its calm anchorage, the best on the west end of Santa Cruz Island, allowed early mariners to interact with populations on the northern Channel Islands as well as mainland Santa Barbara. It became a persistent place that was repeatedly occupied over long periods of time. Early visitors feasted on red abalone, urchins, sea mammals, and other marine delicacies during ceremonies, whether for mourning (Hull 2014) or annual rites such as the winter solstice. Mortuary rites conveyed the symbolic power of the place and created a history of events that became part of a mythical and real past. El Montón was repeatedly visited, modified, and (re)interpreted as social relationships were reinforced. Over time, the mound, whether purposely built higher or not, became more prominent and visible. Leaders with ritual power, wealth, distinction, and exotic goods emerged as social inequality increased.

El Montón clearly fits the characterizations of persistent places as discussed by Schlanger (1992) and Thompson (2010): (1) its location is near easily accessible concentrations of significant resources; (2) the natural features at the site (i.e., the anchorage, situation on a low mound) made it desirable for repeated use; and (3) it was created through practice over an extended time period. El Montón is similar to sites in the southeast, Australia, Brazil, and central California in the prominence of mortuary features, some of which may have been critical in the founding of persistent places.

\section{Conclusion}

By situating investigations of mounds like El Montón within a broader interpretive lens, we see that the building of mounds, whether intentional or not, is widespread among hunter-gatherers in the world. Features such as terraces, remains of feasting events, dedicated cemeteries, and remains of ritual events are well documented. Some mounds, such as El Montón, are thousands of years old, persistent places recognized for millennia. Whether scholars view these as monumental or not, their mere presence is significant in (re)interpretations of hunter-gather societies as more than simple foragers in an evolutionary schema that leads to sedentary agriculturalists. By taking a historical perspective, we see early practices of Chumash Indians that developed in situ for thousands of years. In interpreting the archaeological evidence at El Montón, it is difficult to tease out the ritual from everyday life. Fowles (2013) reminds us that separating secularism from religion reflects a Western perspective, a position that can significantly differ from those of premodern people. Daily practice was probably intimately tied to ritual events associated with ceremonies or religious beliefs in the past, obfuscating the identification of secular versus religion.

The massive mound at El Montón on the west end of Santa Cruz Island can be interpreted in multiple ways. Clearly much of the mound consists of the refuse of meals - fragments of shells and bones deposited for thousands of years. The relatively shallow house depressions attest to people living at the site and hundreds of burials suggest many died there. They had children, and hunted, gathered, and fished for a living. Diets shifted with changes in climate, as seasurface temperatures warmed and cooled during repetitive El Niño-Southern Oscillation (ENSO) cycles. Longer-term climatic events too, such as extended droughts and wet-periods, challenged the Chumash who lived there for millennia. All these issues are significant, particularly with the challenges that we face now and in the upcoming 
centuries, but there is more to El Montón than adaptation to changing environments. As noted in the beginning of this paper, archaeologists throughout the world are excavating and analyzing shell mounds in unique ways. I hope this paper inspires archaeologists working in southern California to contemplate these intellectual trends and think about shell middens in a broader theoretical and cultural context.

Acknowledgments. The project was funded by the WennerGren Foundation for Anthropological Research, the National Geographic Society, UC Santa Barbara (UCSB) ISBER Social Science Research Grants Programs, UCSB Academic Senate Faculty Research Grants, and the UCSB Department of Anthropology. I especially thank Lyndal Laughrin of the Natural Reserve System and David Dewey of the Nature Conservancy for logistical support, and Macduff Everton and Dan Van Dorn for photography and graphics, respectively. I am indebted to many students, particularly Brian Barbier, Erin Bornemann, Tsim Schneider, Peter Nelson, Kristina Gill, Glenn Russell, and Hugh Radde. I especially thank Patricia Chirinos Ogata, who translated the abstract, and the Chumash Indians, including Gil Unzueta, Julie TumamaitStenslie, and the Santa Ynez Band of Chumash. I also acknowledge Tim Pauketat, Susan Alt, Mike Glassow, Greg Wilson, Jon Erlandson, and Amy Gusick for insights in the field. Natasha Johnson and Mari Lyn Salvadore assisted at the Phoebe Hearst of Museum of Anthropology and John Johnson, Jan Timbrook, and Ray Corbett at the Santa Barbara Museum of Natural History. I thank Eric Wohlgemuth for analysis of archaeobotanical remains and Tom Wake for analysis of faunal remains. I also thank Glenn Russell, Tom Blackburn, Brian Barbier, Erin Bornemann, Hugh Radde, Doug Steiderwald, and three anonymous reviewers who commented on drafts. Finally, I am grateful to Robert Kelly for assistance in improving this paper.

Data Availability Statement. Field Notes from Gamble and Wilcoxon's excavations are on file at the UCSB Repository for Archaeological and Ethnographic Collections. Collections and notes from the cemeteries are at the Phoebe Hearst of Museum of Anthropology and the Santa Barbara Museum of Natural History.

\section{References Cited}

Applegate, Richard

1978 'Atishwin: The Dream Helper in South-Central California. Ballena Press, Socorro, California.

Arnold, Bettina

2006 Gender and Archaeological Mortuary Analysis. In Women in Antiquity: Theoretical Approaches to Gender and Archaeology, edited by Sarah M. Nelson, pp. 107140. AltaMira Press, Walnut Creek, California.

Arnold, Jeanne E.

1992 Complex Hunter-Gatherer-Fishers of Prehistoric California: Chiefs, Specialists, and Maritime Adapta- tions of the Channel Islands. American Antiquity 57:6084.

Bailey, Geoffrey N., and Nicholas C. Flemming

2008 Archaeology of the Continental Shelf: Marine Resources, Submerged Landscapes, and Underwater Archaeology. Quaternary Science Reviews 27:21532165.

Basso, Keith H.

1996 Wisdom Sits in Places: Notes on a Western Apache Landscape. In Senses of Place, edited by Steven Feld and Keith H. Basso, pp. 53-90. School of American Research Press, Santa Fe, New Mexico.

Baxter, Jane E.

2008 The Archaeology of Childhood. Annual Review of Anthropology 37:159-175.

Bell, Catherine

1992 Ritual Theory, Ritual Practice. Oxford University Press, New York.

Bourdieu, Pierre

1977 Outline of a Theory of Practice. Cambridge University Press, Cambridge.

Braje, Todd J., and Jon M. Erlandson

2016 California's Red Abalone (Haliotis rufescens) Middens: Comment on Glassow. American Antiquity 81:591-592.

Braje, Todd J., Jon M. Erlandson, and Torben C. Rick

2014 Factors Influencing the Formation of Large Shell Mounds on California's Santa Barbara Channel Region. In The Cultural Dynamics of Shell-Matrix Sites, edited by Mirjana Roksandic, Sheila Mendonça de Souza, Sabine Eggers, Meghan Burchell, and Daniela Klokler, pp. 1-10. University of New Mexico Press, Albuquerque.

Braje, Todd J., Jon M. Erlandson, Torben C. Rick, Paul K. Dayton, and Marco B. Hatch

2009 Fishing from Past to Present: Continuity and Resilience of Red Abalone Fisheries on the Channel Islands, California. Ecological Applications 19:906919.

Breschini, Gary S., Trudy Haversat, and Jon M. Erlandson 1996 California Radiocarbon Dates. 8th ed. Coyote Press, Salinas, California.

Brockwell, Sally

2006 Earth Mounds in Northern Australia: A Review. Australian Archaeology 63:47-56.

Climo, Jacob J., and Maria G. Cattell (editors)

2002 Social Memory and History: Anthropological Perspectives. Altamira Press, Walnut Creek, California.

Cribb, Roger

1991 Getting into a Flap about Shell Mounds in Northern Australia: A Reply to Stone. Archaeology in Oceania 26:23-25.

David, Bruno, and Mura Badulgal

2006 What Happened in Torres Strait 400 Years Ago? Ritual Transformations in an Island Seascape. Journal of Island and Coastal Archaeology 1:123-143.

Dietler, Michael, and Brian Hayden

2001 Digesting the Feast: Good to Eat, Good to Drink, Good to Think: An Introduction. In Feasts: Archaeological and Ethnographic Perspectives on Food, Politics, and Power, edited by Michael Dietler and Brian Hayden, pp. 1-20. Smithsonian Institution Press, Washington, DC.

Ekengren, Fredrick

2013 Contextualising Grave Goods: Theoretical Perspectives and Methodological Implications. In The Oxford 
Handbook of the Archaeology of Death and Burial, edited by Liv Nilsson Stutz and Sarah Tarlow, pp. 173192. Oxford University Press, Oxford.

Erlandson, Jon M.

1994 Early Hunter-Gatherers of the California Coast. Plenum Press, New York.

Fowles, Severin M.

2013 An Archaeology of Doings: Secularism and the Study of Pueblo Religion. School for Advanced Research Press, Santa Fe, New Mexico.

Gamble, Lynn H.

1995 Chumash Architecture: Sweatlodges and Houses. Journal of California and Great Basin Anthropology 17:54-92.

2008 The Chumash at European Contact: Power, Trade, and Feasting Among Complex Hunter-Gatherers. University of California Press, Berkeley.

Gamble, Lynn H., and Brian J. Barbier

2015 Household Archaeology on the Northern Channel Islands of the Santa Barbara Coast, California. Paper presented at the 80th Annual Meeting of the Society for American Archaeology, San Francisco.

Gamble, Lynn H., and Scott Mattingly

2012 Pine Nut Processing in Southern California: Is the Absence of Evidence the Evidence of Absence? American Antiquity 77:263-278.

Gamble, Lynn H., and Alexander R. Simms

2016 The Use of Ground Penetrating Radar, Geomorphological Analysis, Stratigraphy, and Radiocarbon Dating to Identify an Early Period Socially Constructed Landscape on Western Santa Cruz Island. Paper presented at the 50th Annual Meeting of the Society for California Archaeology, Ontario.

Gamble, Lynn H., Phillip L. Walker, and Glenn S. Russell

2001 An Integrative Approach to Mortuary Analysis: Social and Symbolic Dimensions of Chumash Burial Practices. American Antiquity 66:185-212.

Gamble, Lynn H., and Michael Wilken

2008 Kumeyaay Cultural Landscapes of Baja California's Tijuana River Watershed. Journal of California and Great Basin Anthropology. 28:127-151.

Gaspar, Maria Dulce, Paulo DeBlasis, Suzanne K Fish, and Paul R. Fish

2008 Sambaqui (Shell Mound) Societies of Coastal Brazil. In The Handbook of South American Archaeology, edited by H. Silverman and W. Isbell, pp. 319-335. Springer, New York.

Gill, Kristina M.

2015 Ancient Plant Use and the Importance of Geophytes among the Island Chumash of Santa Cruz Island, California. Unpublished Ph.D. dissertation, Department of Anthropology, University of California, Santa Barbara. Glassow, Michael A.

2004 Identifying Complexity during the Early Prehistory of Santa Cruz Island, California. In Foundations of Chumash Complexity, edited by Jeanne, E. Arnold, pp. 17-24. University of California, Cotsen Institute of Archaeology, Los Angeles.

2005 Dolphin Hunting on Santa Cruz Island, California. In The Exploitation and Cultural Importance of Sea Mammals, edited by Gregory G. Monks, pp. 107-120. Oxbow Books, Oxford.

2015 Chronology of Red Abalone Middens on Santa Cruz Island, California, and Evidence for Subsistence and Settlement Change. American Antiquity 80:745759.
2016 Reply to Braje and Erlandson. American Antiquity 81:593-594.

Glassow, Michael A., Lynn H. Gamble, Jennifer E. Perry, and Glenn S. Russell

2007 Prehistory of the Northern California Bight and the Adjacent Transverse Ranges. In California Prehistory: Colonization, Culture, and Complexity, edited by Terry L. Jones and Kathryn A. Klar, pp. 191-213. AltaMira Press, Lanham, Maryland.

Golla, Victor

2011 California Indian Languages. University of California Press, Berkeley.

Grier, Colin

2014 Landscape Construction, Ownership and Social Change in the Southern Gulf Islands of British Columbia. Canadian Journal of Archaeology 38:211249.

Hausmann, Niklas, and Matthew Meredith-Williams

2016 Exploring Accumulation Rates of Shell Deposits through Seasonality Data. Journal of Archaeological Method and Theory, 1-20. DOI: 10.1007/s10816-0169287-x, accessed April 11, 2017.

Hayden, Brian

2001 Fabulous Feasts: A Prolegomenon to the Importance of Feasting. In Feasts: Archaeological and Ethnographic Perspectives on Food, Politics, and Power, edited by Michael Dietler and Brian Hayden, pp. 2364. Smithsonian Institution Press, Washington, DC.

2009 Funerals as Feasts: Why Are They So Important? Cambridge Archaeological Journal 19(1):29-52.

Hull, Kathleen L.

2014 Ritual as Performance in Small-Scale Societies. World Archaeology 46:164-177.

Ingold, Tim

1993 The Temporality of the Landscape. World Archaeology 25:152-174.

Jazwa, Christopher S., Lynn H. Gamble, and Douglas J. Kennett

2013 A High-Precision Chronology for Two House Features at an Early Village Site on Western Santa Cruz Island, California, USA. Radiocarbon 55:185-199.

Jerardino, Antonieta

2010 Large Shell Middens in Lamberts Bay, South Africa: A Case of Hunter-Gatherer Resource Intensification. Journal of Archaeological Science 37:2291-2302.

Johnson, John J.

2000 Social Responses to Climate Change among the Chumash Indians of South-Central California. In The Way the Wind Blows: Climate, History, and Human Action, edited by Roderick J. McIntosh, Joseph A. Tainter, and Susan Keech McIntosh, pp. 301-327. Columbia University Press, New York.

Johnson, John R., and Joseph G. Lorenz

2006 Genetics, Linguistics, and Prehistoric Migrations: An Analysis of California Indian Mitochondrial DNA Lineages. Journal of California and Great Basin Anthropology 26:33-54.

Kamp, Kathryn A.

2001 Where Have All the Children Gone? The Archaeology of Childhood. Journal of Archaeological Method and Theory 8:1-34.

Kennett, Douglas J.

1998 Behavioral Ecology and the Evolution of HunterGatherer Societies on the Northern Channel Islands, California. Ph.D. dissertation, Department of Anthropology, University of California, Santa Barbara. 
2005 The Island Chumash: Behavioral Ecology of a Maritime Society. University of California Press, Berkeley.

Kennett, Douglas J., and James P. Kennett

2000 Competitive and Cooperative Responses to Climatic Instability in Coastal Southern California. American Antiquity 65:379-395.

Kidder, Tristram R.

2011 Transforming Hunter-Gatherer History at Poverty Point. In Hunter-Gatherer Archaeology as Historical Process, edited by Kenneth E. Sassaman and Donald H. Holly, pp. 95-119. University of Arizona Press, Tucson.

Kidder, Tristram R., and Sarah C. Sherwood

2016 Look to the Earth: the Search for Ritual in the Context of Mound Construction. Archaeological and Anthropological Sciences, 1-23 DOI: 10.1007/s12520016-0369-1, accessed April 11, 2017.

King, Chester D

1990 Evolution of Chumash Society: A Comparative Study of Artifacts Used for Social System Maintenance in the Santa Barbara Channel Region before A.D. 1804. Garland Publishing, New York.

Knapp, A. Bernard, and Wendy Ashmore

1999 Archaeological Landscapes: Constructed, Conceptualized, Ideational. In Archaeologies of Landscape: Contemporary Perspective, edited by Wendy Ashmore and A. Bernard Knapp, pp. 1-30. Blackwell Publishers, Malden, Massachusetts.

Landazuri, Heather A.

2015 Analysis of Shellfish Remains from Santa Cruz Island: An Early Period Site Dating from 6,000-2,500 BP. Undergraduate Senior Honors thesis, Department of Anthropology, University of California, Santa Barbara.

Lightfoot, Kent G.

1997 Cultural Construction of Coastal Landscapes: A Middle Holocene Perspective from San Francisco Bay. In Archaeology of the California Coast during the Middle Holocene, edited by Jon M. Erlandson and Michael A. Glassow, pp. 129-141. University of California, Cotsen Institute of Archaeology, Los Angeles.

Lightfoot, Kent G., and Edward M. Luby

2002 Late Holocene in the San Francisco Bay Area: Temporal Trends in the Use and Abandonment of Shell Mounds in the East Bay. In Catalysts to Complexity: Late Holocene Societies of the California Coast, edited by Jon Erlandson and Terry L. Jones, pp. 263-281. University of California, Cotsen Institute of Archaeology, Los Angeles.

Lillehammer, Grete

2010 Archaeology of Children. Complutum 21(2):15-45.

Littleton, Judith, and Harry Allen

2007 Hunter-Gatherer Burials and the Creation of Persistent Places in Southeastern Australia. Journal of Anthropological Archaeology 26:283-298.

Luby, Edward M., and Mark F. Gruber

1999 The Dead Must Be Fed: Symbolic Meanings of the Shell Mounds of the San Francisco Bay Area. Cambridge Archaeological Journal 9:95-108.

McNiven, Ian J.

2012 Ritualized Middening Practices. Journal of Archaeological Method and Theory (20):552-587.

Marquardt, William $\mathrm{H}$.

2010 Shell Mounds in the Southeast: Middens, Monuments, Temple Mounds, Rings, or Works? American Antiquity 75:551-570.
Mathews, Darcy Lane

2014 Funerary Ritual, Ancestral Presence, and the Rocky Point Ways of Death. Ph.D. dissertation, Department of Anthropology, University of Victoria.

Meskell, Lynn

2003 Memory's Materiality: Ancestral Presence, Commemorative Practice and Disjunctive Locales. In Archaeologies of Memory, edited by Ruth Van Dyke and Susan E. Alcock, pp. 34-55. Blackwell Publishers, Malden, Massachusetts.

2007 Back to the Future: From the Past in the Present to the Past in the Past. In Negotiating the Past in the Past, edited by Norman Yoffee, pp. 215-226. University of Arizona Press, Tucson.

Mills, Barbara J., and William H. Walker

2008 Introduction: Memory, Materiality, and Depositional Practice. In Memory Work: Archaeologies of Material Practices, edited by Barbara, J. Mills and William H. Walker, pp. 3-23. School of American Research Press, Santa Fe, New Mexico.

Monroe, Cara, Silvia Gonzalez, John Johnson, Robert Kruszynski, and Brian, M. Kemp

2010 Ancient DNA Analysis of Prehistoric Burials from the Santa Barbara Channel Islands. Paper presented at the 11th Bi National Meeting of Balances and Perspectives on Anthropology and History of Baja California. Ensenada, Baja California.

Moore, Christopher R.

2015 Hunter-Gatherer Histories: The Role of Events in the Construction of the Chiggerville Shell Midden. In The Enigma of the Event: Moments of Consequence in the Ancient Southeast, edited by Jason M. O'Donoughue and Zackary I. Gilmore, pp. 62-76. University of Alabama Press, Tuscaloosa.

Moore, Jerry

2004 The Social Basis of Sacred Space in the Prehispanic Andes: Ritual Landscapes of the Dead in Chimú and Inka Societies. Journal of Archaeological Method and Theory 11:83-124.

Noah, Anna

2005 Household Economies: The Role of Animals in a Historic Period Chiefdom on the California Coast. Ph.D. dissertation, Department of Anthropology, University of California, Los Angeles.

Okada, Hiroaki

1998 Maritime Adaptations in Northern Japan. Arctic Anthropology 35:335-339.

Olson, Ronald L.

1930 Chumash Prehistory. University of California Publications in American Archaeology and Ethnology 28:122.

Parker Pearson, Mike

2000 The Archaeology of Death and Burial. Texas A\&M University Press, College Station.

Pauketat, Timothy R.

2008 Founders' Cults and the Archaeology of Wa-Kan-da. In Memory Work: Archaeologies of Material Practices, edited by Barbara, J. Mills and William H. Walker, pp. 61-80. School of American Research Press, Santa Fe, New Mexico.

Porcasi, Judith F., and Sherri L. Andrews

2001 Evidence for a Prehistoric Mola mola Fishery on the Southern California Coast. Journal of California and Great Basin Anthropology 23:51-56. 
Randall, Asa R.

2011 Remapping Archaic Social Histories along the St. Johns River in Florida. In Hunter-Gatherer Archaeology as Historical Process, edited by Kenneth E. Sassaman and Donald H. Holly, pp. 120-142. University of Arizona Press, Tucson.

Rogers, David B.

1929 Prehistoric Man of the Santa Barbara Coast, California. Special Publications 1. Santa Barbara Museum of Natural History, Santa Barbara, California.

Russo, Michael

1994 Why We Don't Believe in Archaic Ceremonial Mounds and Why We Should: The Case from Florida. Southeastern Archaeology 13:93-109.

Sassaman, Kenneth E.

2010 Structure and Practice in the Archaic Southeast. In Contemporary Archaeology in Theory: The New Pragmatism, 2nd ed., edited by Robert, W. Preucel and Stephen A. Mrozowski, pp. 170-190. Wiley-Blackwell Publishers, West Sussex.

Sassaman, Kenneth E., and Michael J. Heckenberger

2004 Crossing the Symbolic Rubicon in the Southeast. In Signs of Power: the Rise of Cultural Complexity in the Southeast, edited by Jon L. Gibson and Philip J. Carr, pp. 214-233. University of Alabama Press, Tuscaloosa.

Sassaman, Kenneth E., and Asa R. Randall

2012 Shell Mounds of the Middle St. Johns Basin, Northeast Florida. In Early New World Monumentality, edited by Richard L. Burger and Robert M. Rosenswig, pp. 53-77. University Press of Florida, Gainesville.

Saunders, Joe W., Rolfe D. Mandel, C. Garth Sampson, Charles M. Allen, E. Thurman Allen, Daniel A. Bush, James K. Feathers, Kristin J. Gremillion, C. T. Hallmark, H. Edwin Jackson, Jay K. Johnson, Reca Jones, Roger T. Saucier, Gary L. Stringer, and Malcolm F. Vidrine

2005 Watson Brake, a Middle Archaic Mound Complex in Northeast Louisiana. American Antiquity 70:631-568.

Schlanger, Sarah H.

1992 Recognizing Persistent Places in Anasazi. In Space, Time, and Archaeological Landscapes, edited by Jacqueline Rossignol and LuAnn Wandsnider, pp. 91113. Plenum Press, New York.

Schneider, Tsim

2015 Placing Refuge and the Archaeology of Indigenous Hinterlands in Colonial California. American Antiquity 80:695-713.
Sholts, Sabrina B.

2010 Phenotypic Variation among Ancient Human Crania from the Northern Channel Islands of California: Reconstructing Population History across the Holocene. Unpublished Ph.D. dissertation, Department of Anthropology, University of California, Santa Barbara.

Sofaer Derevenski, Joanna

1994 Where are the Children? Accessing Children in the Past. Archaeological Review from Cambridge 13(2):720.

Thompson, Victor D.

2010 The Rhythms of Space-time and the Making of Monuments and Places during the Archaic. In Trend, Tradition, and Turmoil: What Happened to the Southeastern Archaic, edited by David Hurst Thomas and Matthew C. Sangar, pp. 217-227. American Museum of Natural History, New York.

Thompson, Victor D., and C. Fred T. Andrus

2011 Evaluating Mobility, Monumentality, and Feasting at the Sapelo Island Shell Ring Complex. American Antiquity 76:315-344.

Thompson, Victor D., and Thomas J. Pluckhahn

2010 History, Complex Hunter-Gatherers, and the Mounds and Monuments of Crystal River, Florida, USA: A Geophysical Perspective. Journal of Island and Coastal Archaeology 5:33-51.

Timbrook, Jan

2007 Chumash Ethnobotany: Plant Knowledge among the Chumash People of Southern California. Santa Barbara Museum of Natural History Monographs No. 5, Publications in Anthropology No. 1. Heydey Books, Berkeley, Califnornia.

Wilcoxon, Larry

1993 Subsistence and Site Structure: An Approach for Deriving Cultural Information from Coastal Shell Middens. In Archaeology of the Northern Channel Islands of California, edited by Michael A. Glassow, pp. 137-150. Coyote Press, Salinas, California.

Yoffee, Norman

2007 Peering into the Palimpsest. In Negotiating the Past in the Past, edited by Norman Yoffee, pp. 1-9. University of Arizona Press, Tucson.

Submitted August 29, 2016; Revised December 23, 2016; Accepted December 24, 2016 